\title{
SHARP STABILITY OF SOME SPECTRAL INEQUALITIES
}

\author{
LORENZO BRASCO AND ALDO PRATELLI
}

\begin{abstract}
In this work we review two classical isoperimetric inequalities involving eigenvalues of the Laplacian, both with Dirichlet and Neumann boundary conditions. The first one is classically attribuited to Krahn and P. Szego and asserts that among sets of given measure, the disjoint union of two balls with the same radius minimizes the second eigenvalue of the Dirichlet-Laplacian, while the second one is due to G. Szegö and Weinberger and deals with the maximization of the first non trivial eigenvalue of the Neumann-Laplacian. New stability estimates are provided for both of them.
\end{abstract}

\section{INTRODUCTION}

It is generally known that "isoperimetric problems" are very interesting and easy to state, but hard to solve. The fundamental example (from which the name comes) is the classical isoperimetric inequality, asserting that balls have maximal measure among sets of given perimeter, that is,

$$
|\Omega| \leq|B|,
$$

$B$ being a ball of the same perimeter as $\Omega$. Moreover, equality holds if and only if $\Omega$ is a ball. Observe that the homogeneity of the problem automatically implies that the optimization problem has to be posed with some constraints, for example a perimeter constraint as in this case, otherwise the extremum problem is always trivial. On the other hand, one can decide to give up the constraints by adimensionalizing the problem: for example, noticing that

$$
\mathcal{F}(\Omega)=|\Omega|^{(1-N) / N} P(\Omega),
$$

is scaling invariant, the classical isoperimetric statement is equivalent to

$$
|\Omega|^{(1-N) / N} P(\Omega) \geq|B|^{(1-N) / N} P(B),
$$

where $B$ is any ball, with no need for any constraint: this is the most familiar and compact version of the classical isoperimetric property of balls.

Apart from this result linking measure and perimeter, there are a lot of other interesting isoperimetric statements, depending on the choice of the set function $\mathcal{F}$ and of the admissible sets: among these, some fascinating ones are those regarding the spectrum of a given

2010 Mathematics Subject Classification. 47A75; 49Q20; 49R05.

Key words and phrases. Stability for eigenvalues; Krahn-Szego inequality; Szegö-Weinberger inequality; Isoperimetric inequalities. 
elliptic operator, with homogeneous boundary conditions (Dirichlet or Neumann, for example). In the case of the Laplacian operator $-\Delta$, the celebrated Faber-Krahn inequality (see [8, Chapter 3]) asserts

$$
\lambda_{1}(\Omega) \geq \lambda_{1}(B),
$$

where $B$ is a ball with $|B|=|\Omega|$ and $\lambda_{1}$ is the first eigenvalue of the Laplacian with Dirichlet conditions. Moreover, in (1.1) we can have equality only for balls (up to sets of capacity zero). Again, we could drop the measure constraint, by considering the scaling invariant set function $\mathcal{F}(\Omega)=|\Omega|^{2 / N} \lambda_{1}(\Omega)$, so that the previous inequality can be recast into

$$
|\Omega|^{2 / N} \lambda_{1}(\Omega) \geq|B|^{2 / N} \lambda_{1}(B),
$$

where $B$ is any ball and equality holds if and only $\Omega$ coincides with a ball. Also in this case, we have a quantity which is minimized by a certain set, together with a uniqueness statement, which means that strict inequality holds each time the set is not the optimal one. Therefore, it would be of great interest to improve the inequality with a remainder term, that is, finding some meaningful set function $S(\Omega)$, vanishing only for the balls, such that

$$
|\Omega|^{2 / N} \lambda_{1}(\Omega) \geq|B|^{2 / N} \lambda_{1}(B)(1+S(\Omega)) .
$$

The function $S$ must measure (in a suitable sense) the distance of a set from the minimizers: if this can be done, then we have a stability estimate for our isoperimetric statement, that is, a quantitative information on how much a certain set is far from being the optimal one, in terms of how far it is from realizing the equality.

For example, in the two cases considered above, stability estimates have been provided by the second author together with Fusco and Maggi: namely, they have proven

$$
|\Omega|^{(1-N) / N} P(\Omega) \geq|B|^{(1-N) / N} P(B)\left\{1+\alpha_{N} \mathcal{A}(\Omega)^{2}\right\},
$$

and

$$
|\Omega|^{2 / N} \lambda_{1}(\Omega) \geq|B|^{2 / N} \lambda_{1}(B)\left\{1+\gamma_{N} \mathcal{A}(\Omega)^{4}\right\},
$$

where $\mathcal{A}(\Omega)$ is the Fraenkel asymmetry of a set, defined as

$$
\mathcal{A}(\Omega)=\inf \left\{\frac{|\Omega \Delta B|}{|\Omega|}: B \text { ball, }|B|=|\Omega|\right\},
$$

and $\alpha_{N}, \gamma_{N}$ are dimensional constants. For more details, historical remarks and a comprehensive bibliography on other stability results for the aforementioned inequalities, the reader is referred to the papers [5] and [6]. Here we only want to stress that inequality (1.2) is sharp, that is, the exponent 2 for the Fraenkel asymmetry is optimal in the decay rate of the isoperimetric deficit, defined as

$$
\frac{|\Omega|^{(1-N) / N} P(\Omega)}{|B|^{(1-N) / N} P(B)}-1
$$

On the contrary, inequality (1.3) is not sharp: for example, in dimension $N=2$ Bhattacharya has provided an estimate with the power 4 replaced by 3 (see [2, Theorem 2.1]). In any case, actually the best exponent should be 2 , as conjectured in [3] on the basis of the expansion in the case of ellipsoids - in fact, in the isoperimetric problems the ellipsoids 
usually show the optimal rate of convergence. Anyway, a proof of (1.3) with the exponent 2 , which would then be sharp, is still missing.

In the very same spirit, the main scope of this paper is to provide sharp stability estimates for the following classical isoperimetric inequalities, involving eigenvalues of the Laplacian:

- Krahn-Szego inequality, asserting that for every open bounded sets $\Omega \subset \mathbb{R}^{N}$ we have

$$
\lambda_{2}(\Omega) \geq \lambda_{2}\left(\Theta_{2}\right)
$$

where $\Theta_{2}$ is the disjoint union of two balls, both having measure $|\Omega| / 2$, with equality holding if and only if $\Omega=\Theta_{2}$;

- Szegö-Weinberger inequality, dealing with the first non trivial eigenvalue of the Laplacian with Neumann boundary condition, stating that this is maximized by the ball, that is,

$$
\mu_{2}(\Omega) \leq \mu_{2}(B),
$$

where $B$ is a ball having the same measure as $\Omega$, and equality holds if and only if $\Omega$ is a ball.

For both inequalities, we will provide the relative quantitative version, where the proper notion of deficit controls a suitable concept of asymmetry.

We stress that the quantitative version of the Szegö-Weinberger inequality we derive here, i.e.,

$$
\mu_{2}(B)-\mu_{2}(\Omega) \geq c_{N} \mathcal{A}(\Omega)^{2}
$$

is sharp: this is one of the main points of interest of the paper. In fact, while the proof of the inequality is not excessively difficult and relies on a simple geometric intuition, the proof of its sharpness is not trivial at all. As we will explain more in details in Section 5, the main source of difficulties is the fact that $\mu_{2}$, for the ball, is a multiple eigenvalue. The latter fact implies that the shape functional $\Omega \mapsto \mu_{2}(\Omega)$ is not differentiable (in the sense of shape derivative, see [9, Chapter 5] for example) near the ball, which is a maximum point. Roughly speaking, this tells that along some "directions" (i.e., for some perturbations of the ball), the difference of the eigenvalues has a non trivial first order expansion, which means that the superdifferential at the maximum point contains a non zero element. This observation provides some sets $\Omega_{\varepsilon}$, suitably converging to $B$, for which

$$
\mu_{2}(B)-\mu_{2}\left(\Omega_{\varepsilon}\right) \approx \mathcal{A}\left(\Omega_{\varepsilon}\right) .
$$

And in fact, one observes this linear rate of convergence in all the simplest examples, for instance in the case of ellipsoids (which usually, as pointed out above, converge with the sharp convergence rate). Hence, one is easily lead to guess that the right exponent should be 1. To exclude that this is the case and prove the sharpness of the exponent 2 , we will show how to build a suitable family of nearly optimal sets $\Omega_{\varepsilon}$ for which $\mu_{2}(B)-\mu\left(\Omega_{\varepsilon}\right) \approx \mathcal{A}\left(\Omega_{\varepsilon}\right)^{2}$.

On the contrary, concerning the inequality for $\lambda_{2}$, the quantitative version we derive in this work is surely not optimal. Indeed, the proof relies on (1.3) which is not sharp, as already said. However, the key point of our proof of the quantitative Krahn-Szego 
inequality is a Lemma of geometrical content, whose derivation is sharp (see Example 3.4 below).

Besides this, it is interesting to underline that the exponent we obtain for the Fraenkel 2-asymmetry (see below) depends on the dimension: as we will explain in details, this is not a drawback. Indeed, we can show that the optimal exponent has to be dimensiondependent, and in particular has to diverge with the dimension, which is quite surprising, at first glance. In fact, in most examples the optimal exponent does not depend on the dimension.

The paper is organized as follows: in Section 2 we set some of the notations, definitions and results that we will use throughout the paper. We then turn to the first inequality: in Section 3 we provide a stability estimate (Theorem 3.5) for the Krahn-Szego inequality for $\lambda_{2}$. Then in Section 4 we show how to get a quantitative form of the Szegö-Weinberger inequality (Theorem 4.1): this section also includes, as a corollary, a stability estimate for a classical inequality (first conjectured by Kornhauser and Stakgold, [11]) involving $\lambda_{1}$ and $\mu_{2}$. We then turn to the problem of showing that the quantitative estimate of Theorem 4.1 is sharp: we start with Section 5, where we derive the decay rate of the Fraenkel asymmetry for ellipsoids with very small eccentricity, in terms of the Szegö-Weinberger deficit. Surprisingly, ellipsoids turn out not to prescribe the optimal decay rate. Finally, the last section is devoted to the construction of a family of nearly optimals sets for the inequality, whose deficit goes exactly like the Fraenkel asymmetry to the power 2, thus proving the sharpness of Theorem 4.1.

\section{Notations AND PRELIMINARIES}

In what follows, with $N$ we will always denote the dimension. We will also use the convention of indicating with $C_{N}$ (resp., $c_{N}$ ) a sufficiently big (resp., sufficiently small) constant depending on the dimension only, without keeping trace of its precise value, which may increase (resp., decrease) from line to line.

We recall some basic facts about eigenvalues of the Laplace operator for open subsets of $\mathbb{R}^{N}$ : for more details, the reader is referred to the recent monograph [8]. Given an open bounded set $\Omega \subset \mathbb{R}^{N}$, we define its first Dirichlet eigenvalue as

$$
\lambda_{1}(\Omega)=\min _{u \in \mathrm{W}_{0}^{1,2}(\Omega) \backslash\{0\}} \frac{\int_{\Omega}|\nabla u(x)|^{2} d x}{\int_{\Omega}|u(x)|^{2} d x} .
$$

Supposing that $u_{1} \in \mathrm{W}_{0}^{1,2}(\Omega)$ is an eigenfuction corresponding to $\lambda_{1}(\Omega)$, i.e. $u_{1}$ realizes the minimum in the previous Rayleigh quotient, then the second eigenvalue is defined as

$$
\lambda_{2}(\Omega)=\min _{\substack{u \in \mathrm{W}_{0}^{1,2}(\Omega) \backslash\{0\} \\ \int_{\Omega} u(x) u_{1}(x) d x=0}} \frac{\int_{\Omega}|\nabla u(x)|^{2} d x}{\int_{\Omega}|u(x)|^{2} d x},
$$

that is $\lambda_{2}(\Omega)$ is obtained minimizing the same Rayleigh quotient, but now restricting admissible functions to those orthogonal (in the $L^{2}(\Omega)$ sense) to the first eigenfuction. In 
the very same way the successive eigenvalues $\lambda_{k}(\Omega), k \geq 3$, are defined, and we clearly have $\lambda_{1}(\Omega) \leq \lambda_{2}(\Omega) \leq \lambda_{3}(\Omega) \leq \ldots$ In the case that $\Omega$ is not connected, its eigenvalues can be simply obtained by gathering and ordering the eigenvalues of its connected components.

We recall that a first eigenfunction has to be of constant sign in $\Omega$ : this implies, due to the orthogonality condition, that a second eigenfunction $u_{2}$ has to change sign in $\Omega$ (if $\Omega$ is connected) or must have a support disjoint from that of $u_{1}$. We call nodal sets of a certain eigenfunction $u_{k}$ the connected components of the sets

$$
\left\{x \in \Omega: u_{k}>0\right\} \text { and }\left\{x \in \Omega: u_{k}<0\right\},
$$

respectively. Incidentally, we observe that for the second eigenvalue, these nodal domains are known to be two, at most (this is a consequence of the so called Courant-Hilbert nodal domains Theorem, for which the reader is referred to [4]).

It is easily seen that $\lambda_{k}(\Omega)$ scales as a length to the power -2 , that is $\lambda_{k}(t \Omega)=t^{-2} \lambda_{k}(\Omega)$, for every $t>0$, so that usually it is useful to consider the scaling invariant quantity $|\Omega|^{2 / N} \lambda_{k}(\Omega)$. In this way the well-known Faber-Krahn inequality asserts

$$
|\Omega|^{2 / N} \lambda_{1}(\Omega) \geq|B|^{2 / N} \lambda_{1}(B),
$$

where $B$ is any ball. One tool that we will use in this work, is a quantitative version of this isoperimetric statement, which can be rephrased as follows

$$
F K(\Omega) \geq \gamma_{N} \mathcal{A}(\Omega)^{4},
$$

where $F K(\Omega)$ is the Faber-Krahn deficit, defined as

$$
F K(\Omega):=\frac{|\Omega|^{2 / N} \lambda_{1}(\Omega)}{|B|^{2 / N} \lambda_{1}(B)}-1 .
$$

Since the optimal object for $\lambda_{2}$ is the disjoint union of two balls (see below), it is useful to introduce, besides the usual Fraenkel asymmetry, the Fraenkel 2-asymmetry, defined as

$$
\mathcal{A}_{2}(\Omega)=\inf \left\{\frac{\left|\Omega \Delta\left(B_{1} \cup B_{2}\right)\right|}{|\Omega|}:\left|B_{1} \cap B_{2}\right|=0 \text { and }\left|B_{1}\right|=\left|B_{2}\right|=\frac{|\Omega|}{2}\right\},
$$

which measures the distance of a set $\Omega$ from the disjoint union of two balls with the same radius.

Finally, in the case of homogeneous Neumann boundary conditions, the first eigenvalue $\mu_{1}(\Omega)$ is always 0 and corresponds to constant functions, while the first non trivial eigenvalue, if $\Omega$ is a connected open set with Lipschitz boundary, can be defined as

$$
\mu_{2}(\Omega)=\min _{\substack{u \in \mathrm{W}^{1,2}(\Omega) \\ \int_{\Omega} u(x) d x=0}} \frac{\int_{\Omega}|\nabla u(x)|^{2} d x}{\int_{\Omega}|u(x)|^{2} d x} .
$$

The successive eigenvalues can be defined similarly, that is $\mu_{k}(\Omega)$ is obtained by minimizing the same quotient, among functions orthogonal (in the $L^{2}(\Omega)$ sense, again) to the first $k-1$ eigenfunctions. Clearly, if $\Omega$ has $k$ connected components, we have $\mu_{1}(\Omega)=\cdots=$ $\mu_{k}(\Omega)=0$, with corresponding eigenfunctions given by constant function on each connected 
component. Neumann eigenvalues have the same scaling properties as the Dirichlet ones and there holds the Szegö-Weinberger inequality

$$
|\Omega|^{2 / N} \mu_{2}(\Omega) \leq|B|^{2 / N} \mu_{2}(B),
$$

and in this case the ball is the unique maximizer.

For a ball $B$ of radius $r, \mu_{2}(B)$ has multiplicity $N$, that is, $\mu_{2}(B)=\cdots=\mu_{N+1}(B)$, and it can be explicitely computed, together with its corresponding eigenfunctions. Indeed, these are given by (see [1])

$$
\xi_{i}(x):=|x|^{1-\frac{N}{2}} J_{N / 2}\left(\frac{\beta_{N / 2,1}|x|}{r}\right) \frac{x_{i}}{|x|}, i=1, \ldots, N,
$$

where $J_{N / 2}$ is a Bessel function of the first kind, solving the ODE

$$
g^{\prime \prime}(t)+\frac{1}{t} g^{\prime}(t)+\left(1-\frac{N}{2} \frac{1}{t^{2}}\right) g(t)=0
$$

while $\beta_{N / 2,1}$ denotes the first positive zero of the derivative of $t \mapsto t^{1-N / 2} J_{N / 2}(t)$, i.e. it verifies

$$
\beta_{N / 2,1} J_{N / 2,1}^{\prime}\left(\beta_{N / 2,1}\right)+\left(1-\frac{N}{2}\right) J_{N / 2}\left(\beta_{N / 2,1}\right)=0
$$

Observe in particular that the radial part of $\xi_{i}$, given by

$$
\varphi_{N}(|x|):=|x|^{1-\frac{N}{2}} J_{N / 2}\left(\frac{\beta_{N / 2,1}|x|}{r}\right)
$$

satisfies the ODE (of Bessel type)

$$
g^{\prime \prime}(t)+\frac{N-1}{t} g^{\prime}(t)+\left(\mu_{2}(B)-\frac{N-1}{t^{2}}\right) g(t)=0,
$$

and one can calculate

$$
\mu_{2}(B)=\frac{\beta_{N / 2,1}^{2}}{r^{2}}
$$

\section{Minimization of the Second eigenvalue of the Dirichlet-Laplacian}

We now recall the classical isoperimetric inequality concerning the second eigenvalue of the Laplacian with homogeneous Dirichlet boundary conditions, a result which is usually attributed to Krahn ([12]) and Peter Szego ${ }^{1}$ (Pólya gave credit to this in [14]). It worths remarking that the same result was also proven independently by Hong (see [10]), exactly in the same years as Szego. We give this result directly in the scaling invariant form, so disregarding the constraint on the measure of the admissible sets.

\footnotetext{
${ }^{1}$ Son of Gabor Szegö. The difference in the spelling of the surname is due to the fact that Peter passed most of his life in the US and consequently "Americanized" his name. We owe these informations to the kind courtsey of Mark S. Ashbaugh.
} 
Krahn-Szego inequality. For every open bounded set $\Omega \subset \mathbb{R}^{N}$, we have

$$
|\Omega|^{2 / N} \lambda_{2}(\Omega) \geq\left|\Theta_{2}\right|^{2 / N} \lambda_{2}\left(\Theta_{2}\right)
$$

where $\Theta_{2}=B_{+} \cup B_{-}$is any disjoint union of two balls having the same radius.

We aim to give a quantitative version of this result: namely, we wish to estimate how far a generic set is from being the disjoint union of two equal balls, in terms of how far the product $|\cdot|^{2 / N} \lambda_{2}(\cdot)$ is from the minimum. Thus, for every bounded open set $\Omega$, we introduce the Krahn-Szego deficit

$$
K S(\Omega):=\frac{|\Omega|^{2 / N} \lambda_{2}(\Omega)}{\left|\Theta_{2}\right|^{2 / N} \lambda_{2}\left(\Theta_{2}\right)}-1 .
$$

We begin with a simple but useful technical Lemma.

Lemma 3.1. Let $\Omega \subset \mathbb{R}^{N}$ be a bounded open set. Then there exist two disjoint sets $\Omega_{+}, \Omega_{-} \subset \Omega$ such that

$$
\lambda_{2}(\Omega)=\max \left\{\lambda_{1}\left(\Omega_{+}\right), \lambda_{1}\left(\Omega_{-}\right)\right\} .
$$

Proof. Let $u_{1}$ and $u_{2}$ be two eigenfunctions relative to the first two eigenvalues $\lambda_{1}(\Omega)$ and $\lambda_{2}(\Omega)$, and let us distinguish two possibilities:

(i) either $u_{2}$ has always the same sign in $\Omega$;

(ii) or $u_{2}$ changes sign on $\Omega$.

In case (i), let us define

$$
\Omega_{+}=\left\{\left|u_{1}\right|>0\right\}, \quad \Omega_{-}=\left\{\left|u_{2}\right|>0\right\} .
$$

Since neither $u_{1}$ nor $u_{2}$ change sign in $\Omega$, by the orthogonality condition

$$
\int_{\Omega} u_{2}(x) u_{1}(x) d x=0
$$

we immediately get that $\Omega_{+}$and $\Omega_{-}$are disconnected. But then, since $u_{2} \in \mathrm{W}_{0}^{1,2}\left(\Omega_{-}\right)$, and since of course

$$
\int_{\Omega} u(x) u_{1}(x) d x=0, \text { for every } u \in \mathrm{W}_{0}^{1,2}\left(\Omega_{-}\right),
$$

we immediately derive that

$$
\lambda_{1}\left(\Omega_{-}\right) \leq \frac{\int_{\Omega}\left|\nabla u_{2}(x)\right|^{2} d x}{\int_{\Omega}\left|u_{2}(x)\right|^{2} d x}=\lambda_{2}(\Omega) \leq \inf _{u \in \mathrm{W}_{0}^{1,2}\left(\Omega_{-}\right)} \frac{\int_{\Omega}|\nabla u(x)|^{2} d x}{\int_{\Omega}|u(x)|^{2} d x}=\lambda_{1}\left(\Omega_{-}\right) .
$$

Hence, we directly get (3.3) simply by noticing, that since $u_{1}$ is supported in $\Omega_{+}$, then

$$
\lambda_{1}\left(\Omega_{+}\right)=\lambda_{1}(\Omega)<\lambda_{2}(\Omega) .
$$


On the other hand, in case (ii), we consider the nodal sets of $u_{2}$

$$
\Omega_{+}=\left\{x \in \Omega: u_{2}(x)>0\right\} \quad \Omega_{-}=\left\{x \in \Omega: u_{2}(x)<0\right\}
$$

and we observe that, in the weak sense, we have

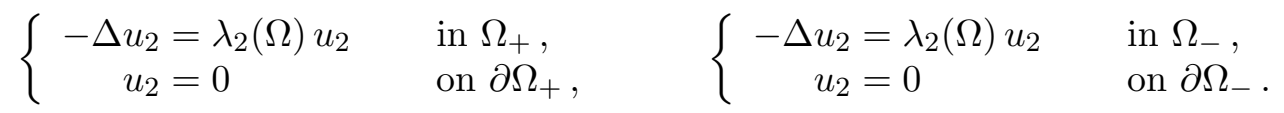

This tells us that $u_{2}$ is an eigenfunction both for $\Omega_{+}$and $\Omega_{-}$, and in particular it is strictly positive in $\Omega_{+}$and strictly negative in $\Omega_{-}$, so it corresponds to the first eigenvalue for both sets. Hence we get

$$
\lambda_{2}(\Omega)=\lambda_{1}\left(\Omega_{+}\right)=\lambda_{1}\left(\Omega_{-}\right),
$$

and (3.3) follows also in this case.

Corollary 3.2 (Disjoint equal balls minimize $\lambda_{2}$ ). For any bounded open set $\Omega \subset \mathbb{R}^{N}$, there holds

$$
\lambda_{2}(\Omega) \geq \lambda_{2}\left(B_{+} \cup B_{-}\right)=\lambda_{1}(B)\left(\frac{2}{|\Omega|}\right)^{2 / N},
$$

being $B_{+}$and $B_{-}$any two disjoint balls with $\left|B_{+}\right|=\left|B_{-}\right|=|\Omega| / 2$, and being $B$ a ball of unit volume. Moreover, the equality holds if and only if $\Omega$ is in fact such a disjoint union (up to a set of capacity zero).

Proof. Let $\Omega_{+}$and $\Omega_{-}$be as in Lemma 3.1 , and let $\widetilde{B}_{+}$and $\widetilde{B}_{-}$be two disjoint balls with $\left|\widetilde{B}_{ \pm}\right|=\left|\Omega_{ \pm}\right|$. By the standard Faber-Krahn inequality we know that

$$
\lambda_{1}\left(\Omega_{+}\right) \geq \lambda_{1}\left(\widetilde{B}_{+}\right), \quad \lambda_{1}\left(\Omega_{-}\right) \geq \lambda_{1}\left(\widetilde{B}_{-}\right),
$$

then by (3.3) we have, calling $B$ the ball of unit volume,

$$
\begin{aligned}
\lambda_{2}(\Omega) & \geq \max \left\{\lambda_{1}\left(\widetilde{B}_{+}\right), \lambda_{1}\left(\widetilde{B}_{-}\right)\right\}=\max \left\{\frac{\lambda_{1}(B)}{\left|\widetilde{B}_{+}\right|^{2 / N}}, \frac{\lambda_{1}(B)}{\left|\widetilde{B}_{-}\right|^{2 / N}}\right\} \\
& =\frac{\lambda_{1}(B)}{\left(\min \left\{\left|\Omega_{+}\right|,\left|\Omega_{-}\right|\right\}\right)^{2 / N}} \geq \frac{\lambda_{1}(B)}{(|\Omega| / 2)^{2 / N}}=\lambda_{2}\left(B_{+} \cup B_{-}\right) .
\end{aligned}
$$

This gives the asserted inequality in (3.4), while the equality comes directly by the scaling properties of $\lambda_{1}$. Moreover, if equality holds in (3.4), then the inequalities (3.5) and (3.6) above must both be equalities. In particular, the fact that the equality holds in (3.5) implies - by Faber-Krahn inequality - that both $\Omega_{+}$and $\Omega_{-}$are balls. On the other hand, the fact that the equality holds in (3.6) implies that $\left|\Omega_{+}\right|=\left|\Omega_{-}\right|=|\Omega| / 2$. This completes the proof.

Keeping the same notations as in Lemma 3.1, the next result tells us that the errors of the two subsets $\Omega_{+}$and $\Omega_{-}$control the distance of $\Omega$ from a minimizer of $\lambda_{2}$, i.e. a disjoint union of two equal balls: this is a crucial fact, preliminary to the main estimate of this section. 
Lemma 3.3. Let $\Omega \subset \mathbb{R}^{N}$ be an open bounded set. With the notations of Lemma 3.1, we have

$$
\mathcal{A}_{2}(\Omega) \leq C_{N}\left(\mathcal{A}\left(\Omega_{+}\right)+\left|\frac{1}{2}-\frac{\left|\Omega_{+}\right|}{|\Omega|}\right|+\mathcal{A}\left(\Omega_{-}\right)+\left|\frac{1}{2}-\frac{\left|\Omega_{-}\right|}{|\Omega|}\right|\right)^{2 /(N+1)},
$$

for a suitable dimensional constant $C_{N}$.

Proof. The idea of the proof is quite easy: all we have to do, is to take a pair of optimal balls for $\mathcal{A}\left(\Omega_{+}\right)$and $\mathcal{A}\left(\Omega_{-}\right)$and to make them admissible for estimating $\mathcal{A}_{2}(\Omega)$, possibly rescaling and translating them. We start setting

$$
\varepsilon_{+}=\frac{1}{2}-\frac{\left|\Omega_{+}\right|}{|\Omega|}, \quad \quad \varepsilon_{-}=\frac{1}{2}-\frac{\left|\Omega_{-}\right|}{|\Omega|},
$$

and let $B_{1}, B_{2}$ be two balls centered at the origin, such that $\left|B_{1}\right|=\left|\Omega_{+}\right|$and $\left|B_{2}\right|=\left|\Omega_{-}\right|$, with

$$
\mathcal{A}\left(\Omega_{+}\right)=\frac{2\left|\left(B_{1}+x_{0}\right) \backslash \Omega_{+}\right|}{\left|\Omega_{+}\right|}, \quad \mathcal{A}\left(\Omega_{-}\right)=\frac{2\left|\left(B_{2}+y_{0}\right) \backslash \Omega_{-}\right|}{\left|\Omega_{-}\right|},
$$

for suitable $x_{0}, y_{0}$. One would like to use directly $\left(B_{1}+x_{0}\right) \cup\left(B_{2}+y_{0}\right)$ as an admissible set for $\mathcal{A}_{2}(\Omega)$, but we can not do it for two reasons: the first is that it could happen that $\left|\left(B_{1}+x_{0}\right) \cap\left(B_{2}+y_{0}\right)\right|>0$; the second is that in general $\left|B_{1}\right| \neq\left|B_{2}\right|$. So first of all we define two new balls rescaling $B_{1}$ and $B_{2}$, that is,

$$
\widetilde{B}_{1}=\left(1-2 \varepsilon_{+}\right)^{-1 / N} B_{1}, \quad \widetilde{B}_{2}=\left(1-2 \varepsilon_{-}\right)^{-1 / N} B_{2},
$$

so that both $\widetilde{B}_{1}$ and $\widetilde{B}_{2}$ have volume $|\Omega| / 2$. We have now to translate them, since it is not guaranteed that the two balls $\widetilde{B}_{1}+x_{0}$ and $\widetilde{B}_{2}+y_{0}$ are disjoint: we make this translation in the direction given by $y_{0}-x_{0}$, so we need to estimate the width of the set $\left(\widetilde{B}_{1}+x_{0}\right) \cap\left(\widetilde{B}_{2}+y_{0}\right)$ in this direction. Calling $\ell$ this quantity, it is not difficult to see that there holds

$$
\ell^{(N+1) / 2}|\Omega|^{(N-1) /(2 N)} \approx C_{N}\left|\left(\widetilde{B}_{1}+x_{0}\right) \cap\left(\widetilde{B}_{2}+y_{0}\right)\right| .
$$

This shows that we need an information on $\left|\left(\widetilde{B}_{1}+x_{0}\right) \cap\left(\widetilde{B}_{2}+y_{0}\right)\right|$. Observe that with simple algebraic manipulations, we have

$$
\left(\widetilde{B}_{1}+x_{0}\right) \cap\left(\widetilde{B}_{2}+y_{0}\right) \subset\left(\left(\widetilde{B}_{1}+x_{0}\right) \backslash \Omega_{+}\right) \cup\left(\left(\widetilde{B}_{2}+y_{0}\right) \backslash \Omega_{-}\right) \cup\left(\Omega_{-} \cap \Omega_{+}\right),
$$

so that using $\left|\Omega_{+} \cap \Omega_{-}\right|=0$, we obtain

$$
\begin{aligned}
\mid\left(\widetilde{B}_{1}+x_{0}\right) \cap\left(\widetilde{B}_{2}\right. & \left.+y_{0}\right)|\leq|\left(\widetilde{B}_{1}+x_{0}\right) \backslash \Omega_{+}|+|\left(\widetilde{B}_{2}+y_{0}\right) \backslash \Omega_{-} \mid \\
& \leq\left|\widetilde{B}_{1} \Delta B_{1}\right|+\left|\left(B_{1}+x_{0}\right) \backslash \Omega_{+}\right|+\left|\widetilde{B}_{2} \Delta B_{2}\right|+\left|\left(B_{2}+x_{0}\right) \backslash \Omega_{-}\right| \\
& \leq C_{N}|\Omega|\left(\left|\varepsilon_{+}\right|+\left|\varepsilon_{-}\right|+\mathcal{A}\left(\Omega_{+}\right)+\mathcal{A}\left(\Omega_{-}\right)\right),
\end{aligned}
$$

where we have used $\left|\widetilde{B}_{1} \Delta B_{1}\right|=|\Omega|\left|\varepsilon_{+}\right|$and $\left|\widetilde{B}_{2} \Delta B_{2}\right|=|\Omega|\left|\varepsilon_{-}\right|$. We now consider the couple of balls $\widetilde{B}_{1}+x_{0}$ and $\widetilde{B}_{2}+\bar{y}_{0}$, with $\bar{y}_{0}$ given by

$$
\bar{y}_{0}=y_{0}+\ell \frac{y_{0}-x_{0}}{\left|y_{0}-x_{0}\right|}
$$


so that these new balls are disjoint. Finally, let us observe that

$$
\left|\left(y_{0}+\widetilde{B}_{2}\right) \Delta\left(\bar{y}_{0}+\widetilde{B}_{2}\right)\right| \approx \ell|\Omega|^{(N-1) / N},
$$

so that

$$
\begin{aligned}
\left|\left(y_{0}+B_{2}\right) \Delta\left(\bar{y}_{0}+\widetilde{B}_{2}\right)\right| & \leq\left|\left(y_{0}+B_{2}\right) \Delta\left(y_{0}+\widetilde{B}_{2}\right)\right|+\left|\left(y_{0}+\widetilde{B}_{2}\right) \Delta\left(\bar{y}_{0}+\widetilde{B}_{2}\right)\right| \\
& \leq|\Omega|\left|\varepsilon_{-}\right|+C_{N} \ell|\Omega|^{(N-1) / N} .
\end{aligned}
$$

Up to now, we have collected all the elements in order to complete the proof: we start observing that

$$
\begin{aligned}
\mathcal{A}_{2}(\Omega) & \leq\left|\Omega \backslash\left(\left(x_{0}+\widetilde{B}_{1}\right) \cup\left(\bar{y}_{0}+\widetilde{B}_{2}\right)\right)\right| \\
& \leq\left|\Omega_{+} \backslash\left(x_{0}+\widetilde{B}_{1}\right)\right|+\left|\Omega_{-} \backslash\left(y_{0}+\widetilde{B}_{2}\right)\right|+\left(|\Omega|-\left|\Omega_{+}\right|-\left|\Omega_{-}\right|\right),
\end{aligned}
$$

then we estimate the three terms above. One has clearly

$$
|\Omega|-\left|\Omega_{+}\right|-\left|\Omega_{-}\right|=|\Omega|\left(\varepsilon_{+}+\varepsilon_{-}\right),
$$

while

$$
\left|\Omega_{+} \backslash\left(x_{0}+\widetilde{B}_{1}\right)\right| \leq\left|\Omega_{+} \backslash\left(x_{0}+B_{1}\right)\right|+\left|\widetilde{B}_{1} \Delta B_{1}\right| \leq|\Omega| \frac{\mathcal{A}\left(\Omega_{+}\right)}{2}+|\Omega|\left|\varepsilon_{+}\right|
$$

and

$$
\begin{aligned}
\left|\Omega_{-} \backslash\left(\bar{y}_{0}+\widetilde{B}_{2}\right)\right| & \leq\left|\Omega_{-} \backslash\left(y_{0}+B_{2}\right)\right|+\left|\left(y_{0}+B_{2}\right) \Delta\left(\bar{y}_{0}+\widetilde{B}_{2}\right)\right| \\
& \leq|\Omega| \frac{\mathcal{A}\left(\Omega_{-}\right)}{2}+|\Omega|\left|\varepsilon_{-}\right|+C_{N} \ell|\Omega|^{(N-1) / N} \\
& \leq|\Omega| \frac{\mathcal{A}\left(\Omega_{-}\right)}{2}+|\Omega|\left|\varepsilon_{-}\right|+C_{N}|\Omega|\left(\left|\varepsilon_{+}\right|+\left|\varepsilon_{-}\right|+\mathcal{A}\left(\Omega_{+}\right)+\mathcal{A}\left(\Omega_{-}\right)\right)^{2 /(N+1)},
\end{aligned}
$$

thanks to (3.10), (3.8) and (3.9). Inserting the last three estimates into (3.11) finally yields (3.7).

It is not difficult to show that the exponent $2 /(N+1)$ in $(3.7)$ is indeed sharp.

Example 3.4. Let us fix a small parameter $\varepsilon>0$ and consider the following set

$$
\Omega^{\varepsilon}=\left\{(x, y):(x+1-\varepsilon)^{2}+y^{2}<1\right\} \cup\left\{(x, y):(x-1+\varepsilon)^{2}+y^{2}<1\right\},
$$

which is just the union of two disks of radius 1 , with an overlapping part whose area is of order $\varepsilon^{3 / 2}$. With the previous notations, we have

$$
\Omega_{+}^{\varepsilon}=\left\{(x, y) \in \Omega^{\varepsilon}: x>0\right\} \quad \text { and } \quad \Omega_{-}^{\varepsilon}=\left\{(x, y) \in \Omega^{\varepsilon}: x<0\right\},
$$

and by symmetry, we can work only with $\Omega_{+}^{\varepsilon}$. It is not difficult to see that $\mathcal{A}\left(\Omega_{+}^{\varepsilon}\right)=O\left(\varepsilon^{3 / 2}\right)$ : indeed, consider a ball $B_{+}$with the same measure as $\Omega_{+}^{\varepsilon}$ and centered at $(1-\varepsilon, 0)$, that is $\pi-\left|B_{+}\right| \simeq \varepsilon^{3 / 2}$, so that the radius $r_{\varepsilon}$ of $B_{+}$is such that $1-r_{\varepsilon} \simeq \varepsilon^{3 / 2}$. We have $\left|\Omega_{+}^{\varepsilon} \backslash B_{+}\right|=c \varepsilon^{3 / 2}$ so that

$$
\mathcal{A}\left(\Omega_{+}^{\varepsilon}\right) \leq c \varepsilon^{3 / 2} .
$$


Translating $B_{+}$if necessary, we can not improve on an estimate of the type $\left|\Omega_{+}^{\varepsilon} \backslash B_{+}\right| \simeq \varepsilon^{3 / 2}$, thus showing that $\mathcal{A}\left(\Omega_{+}^{\varepsilon}\right)=O\left(\varepsilon^{3 / 2}\right)$.

On the contrary, observe that in order to let our ball be admissible for $\mathcal{A}_{2}(\Omega)$, we have to translate it rightward of a length which is of order $\varepsilon$, so to avoid the overlapping region. If we call $\widetilde{B}_{+}$this new ball, we then have $\left|\Omega_{+} \backslash \widetilde{B}_{+}\right| \simeq \varepsilon$, so that

$$
\mathcal{A}_{2}(\Omega)^{3 / 2} \simeq \varepsilon^{3 / 2} \simeq \mathcal{A}\left(\Omega_{+}\right)
$$

The very same computations also apply to the case $N \geq 3$, with the exponent $(N+1) / 2$ in place of $3 / 2$. This shows that the exponent in (3.7) is sharp.

Thanks to the previous geometrical result, we can now give the following stability estimate, which is the main result of this section.

Theorem 3.5. Let $\Omega \subset \mathbb{R}^{N}$ be an open bounded set. Then

$$
K S(\Omega) \geq C_{N} \mathcal{A}_{2}(\Omega)^{2(N+1)}
$$

with the constant $C_{N}$ depending on $N$ only.

Proof. Thanks to Lemma 3.1, we know the existence of two disjoint sets $\Omega_{+}, \Omega_{-} \subset \Omega$ such that (3.3) holds. We then set

$$
\delta_{+}=\left|\Omega_{+}\right|-\frac{|\Omega|}{2}, \quad \delta_{-}=\left|\Omega_{-}\right|-\frac{|\Omega|}{2}
$$

and we observe that it must be $\delta_{+}+\delta_{-} \leq 0$. We will prove the Theorem as soon as we show that

$$
K S(\Omega) \geq \frac{1}{C_{N}} \max \left\{\mathcal{A}\left(\Omega_{+}\right)^{4}+\left|\frac{1}{2}-\frac{\left|\Omega_{+}\right|}{|\Omega|}\right|, \mathcal{A}\left(\Omega_{-}\right)^{4}+\left|\frac{1}{2}-\frac{\left|\Omega_{-}\right|}{|\Omega|}\right|\right\}
$$

thanks to Lemma 3.3. To obtain (3.13), it will be useful to distinguish two cases, namely whether $\delta_{+}$and $\delta_{-}$are both non positive, or they have opposite sign. Observe that since the quantities appearing in the right-hand side of (3.13) are all bounded by a universal constant, it is not restrictive to prove (3.13) under the further assumption

$$
K S(\Omega) \leq 1
$$

Case 1. $\delta_{+}$and $\delta_{-}$are both non-positive.

In this case, let us apply the quantitative Faber-Krahn inequality (2.1) to $\Omega_{+}$. Calling again $B$ the ball of unit volume, and recalling (3.3) and the definition (3.2) of the Krahn-Szego 
deficit, we find

$$
\begin{aligned}
\gamma_{N} \mathcal{A}\left(\Omega_{+}\right)^{4} & \leq \frac{\left|\Omega_{+}\right|^{2 / N} \lambda_{1}\left(\Omega_{+}\right)}{\lambda_{1}(B)}-1 \leq \frac{\left(\frac{|\Omega|}{2}+\delta_{+}\right)^{2 / N} \lambda_{2}(\Omega)}{2^{-2 / N}\left|\Theta_{2}\right|^{2 / N} \lambda_{2}\left(\Theta_{2}\right)}-1 \\
& =\frac{|\Omega|^{2 / N}\left(1+\frac{2 \delta_{+}}{|\Omega|}\right)^{2 / N} \lambda_{2}(\Omega)}{\left|\Theta_{2}\right|^{2 / N} \lambda_{2}\left(\Theta_{2}\right)}-1=(K S(\Omega)+1)\left(1+\frac{2 \delta_{+}}{|\Omega|}\right)^{2 / N}-1 \\
& \leq(K S(\Omega)+1)\left(1+\frac{4}{N} \frac{\delta_{+}}{|\Omega|}\right)-1,
\end{aligned}
$$

which can be rewritten as follows

$$
\gamma_{N} \mathcal{A}\left(\Omega_{+}\right)^{4} \leq K S(\Omega)+\frac{4}{N} \frac{\delta_{+}}{|\Omega|}(K S(\Omega)+1)
$$

Using that $\delta_{+} \leq 0$ and $K S(\Omega) \geq 0$, we thus obtain

$$
K S(\Omega) \geq \gamma_{N} \mathcal{A}\left(\Omega_{+}\right)^{4}+\frac{4}{N}\left(\frac{1}{2}-\frac{\left|\Omega_{+}\right|}{|\Omega|}\right) .
$$

Hence, the same calculations with $\Omega_{-}$in place of $\Omega_{+}$yield (3.13)

Case 2. $\delta_{+}$and $\delta_{-}$have opposite sign.

Let us assume for example that $\delta_{+} \geq 0$ and $\delta_{-} \leq 0$. Hence, we still have the estimate (3.14) for both $\Omega_{+}$and $\Omega_{-}$, but it is no more true that

$$
\left(\frac{1}{2}-\frac{\left|\Omega_{+}\right|}{|\Omega|}\right)=\left|\frac{1}{2}-\frac{\left|\Omega_{+}\right|}{|\Omega|}\right|
$$

However, recalling that $\Omega_{+}$and $\Omega_{-}$are disjoint, we have

$$
\frac{1}{2}-\frac{\left|\Omega_{+}\right|}{|\Omega|} \geq-\left(\frac{1}{2}-\frac{\left|\Omega_{-}\right|}{|\Omega|}\right) \geq-\frac{N}{4} K S(\Omega),
$$

where in the second inequality we used (3.15) applied to $\Omega_{-}$. Therefore, using this information in (3.14)) and using that $K S(\Omega) \geq 1$, we immediately get

$$
K S(\Omega) \geq \frac{\gamma_{N}}{3} \mathcal{A}\left(\Omega_{+}\right)^{4}
$$

while (3.16) can be also rephrased as

$$
\left|\frac{1}{2}-\frac{\left|\Omega_{+}\right|}{|\Omega|}\right|=\frac{\left|\Omega_{+}\right|}{|\Omega|}-\frac{1}{2} \leq \frac{N}{4} K S(\Omega) .
$$

These estimates on $\Omega_{+}$, together with the validity of (3.14) for $\Omega_{-}$and with the fact that $\delta_{-} \leq 0$, ensure that (3.13) holds also in this case.

Concerning the sharpness of estimate (3.12), some remarks are in order. 
Remark 3.6. Let us define

$\kappa:=$ optimal exponent for the quantitative Faber-Krahn inequality,

then observe that our proof actually provides the following estimate

$$
K S(\Omega) \geq C_{N} \mathcal{A}_{2}(\Omega)^{\kappa \cdot(N+1) / 2} .
$$

We stress the fact that the proof of (3.17) consisted of two steps: the first one is the geometrical result of Lemma 3.3, which enables to switch from the error terms of $\Omega_{+}$and $\Omega_{-}$to $\mathcal{A}_{2}(\Omega)$; the second one is the application of the quantitative Faber-Krahn inequality to the two relevant pieces $\Omega_{+}$and $\Omega_{-}$. Both steps are optimal (in the second, the precise value of $\kappa$ actually plays no role), but unfortunately this is of course not a warranty of the sharpness of estimate (3.17).

In the end, we are not able to decide whether the exponent for $\mathcal{A}_{2}$ in (3.17) is optimal or not: in any case, we point out that the optimal exponent for the quantitative Krahn-Szego inequality has to be dimension-dependent. To see this, it is enough to consider the very same set of Example 3.4 and then observe that, keeping the same notations, we have

$$
K S\left(\Omega_{\varepsilon}\right)=F K\left(\Omega_{\varepsilon}^{+}\right) \leq O\left(\mathcal{A}\left(\Omega_{\varepsilon}^{+}\right)\right)=O\left(\mathcal{A}_{2}\left(\Omega_{\varepsilon}\right)^{(N+1) / 2}\right) .
$$

\section{Maximization of the first non trivial eigenvalue of the NEUMANN-LAPLACIAN}

In the case of Neumann conditions, the minimization of the $k$-th eigenvalue is no more interesting, since we always have $\mu_{k}(\Omega)=0$ for any set with $k$ connected components. On the contrary, the maximization problem with a measure constraint becomes interesting. The extremal set is again known to be a ball.

Szegö-Weinberger inequality. For every open bounded set $\Omega \subset \mathbb{R}^{N}$ there holds

$$
|B|^{2 / N} \mu_{2}(B) \geq|\Omega|^{2 / N} \mu_{2}(\Omega),
$$

where $B$ is any ball. Moreover, equality holds if and only if $\Omega$ itself is a ball.

In order to provide a quantitative estimate for this inequality, it is better to have an idea of how the classical proof goes on: the arguments below are due to Weinberger (see [17]), while Gabor Szegö's proof relies on conformal mappings and thus it is only valid when $N=2$ (see [15]).

Roughly speaking, given a domain $\Omega$, the fact that the membrane is free on the boundary (that is, we have no Dirichlet conditions) allows to use directly the eigenfunctions of the ball in the Rayleigh quotient definining $\mu_{2}(\Omega)$, then giving the desired estimate, as far as $\mu_{2}(\Omega)$ is defined as the minimum of this quotient. The only point which requires some attention, is the fact that the zero-mean condition on $\Omega$ is in general not satisfied by the eigenfunctions of the ball, so they would not be admissible: anyway, it is just a matter of properly choosing the origin. 
After having given the idea, let us start with the proof: we denote with $B$ the ball centered at the origin, having radius given by

$$
r=\left(\frac{|\Omega|}{\omega_{N}}\right)^{\frac{1}{N}}
$$

where $\omega_{N}$ denotes the measure of the unitary ball, so that $|B|=|\Omega|$. With the same notations as in Section 2, the eigenfunctions of $B$ corresponding to $\mu_{2}(B)$ are given by

$$
\xi_{i}=\varphi_{N}(|x|) \frac{x_{i}}{|x|}, \quad i=1, \ldots, N .
$$

It is important to stress here that $\varphi_{N}(0)=0, \varphi_{N}$ is strictly increasing in $(0, r)$, and $\varphi_{N}^{\prime}(r)=0$. In order to make $\xi_{i}$ admissible for the variational problem defining $\mu_{2}(\Omega)$, we extend continuously $\varphi_{N}$ as follows

$$
\phi_{N}(t)=\left\{\begin{array}{l}
\varphi_{N}(t), \quad t \in[0, r] \\
\varphi_{N}(r), \quad t \in(r, \infty)
\end{array}\right.
$$

and then we consider the new functions

$$
\Xi_{i}(x)=\phi_{N}(|x|) \frac{x_{i}}{|x|}, \quad \quad i=1, \ldots, N .
$$

It is now possible to choose the origin of the coordinate axes in such a way that $\int_{\Omega} \Xi_{i}(x) d x=$ $0, i=1, \ldots, N$ (see [8, Lemma 6.2.2]), hence we infer

$$
\mu_{2}(\Omega) \leq \frac{\int_{\Omega}\left|\nabla \Xi_{i}(x)\right|^{2} d x}{\int_{\Omega} \Xi_{i}(x)^{2} d x}, \quad i=1, \ldots, N .
$$

Now, a summation over $i=1, \ldots, N$ yields

$$
\mu_{2}(\Omega) \leq \frac{\sum_{i=1}^{N} \int_{\Omega}\left|\nabla \Xi_{i}(x)\right|^{2} d x}{\sum_{i=1}^{N} \int_{\Omega} \Xi_{i}^{2}(x) d x} .
$$

This trick is essential in order to let the angular variables disappear: indeed, while the denominator can be immediately written as

$$
\sum_{i=1}^{N} \int_{\Omega} \Xi_{i}(x)^{2} d x=\int_{\Omega} \phi_{N}(|x|)^{2} d x,
$$

computing the gradient of $\Xi_{i}$ there is a cancellation of the mixed term $\phi_{N} \phi_{N}^{\prime}$, and one simply gets

$$
\left|\nabla \Xi_{i}\right|^{2}=\phi_{N}^{\prime}(|x|)^{2} \frac{x_{i}^{2}}{|x|^{2}}+\frac{\phi_{N}(|x|)^{2}}{|x|^{2}}\left(1-\frac{x_{i}^{2}}{|x|^{2}}\right)
$$


Summing all up, we have obtained

$$
\mu_{2}(\Omega) \leq \frac{\int_{\Omega} \phi_{N}^{\prime}(|x|)^{2}+(N-1) \frac{\phi_{N}(|x|)^{2}}{|x|^{2}} d x}{\int_{\Omega} \phi_{N}(|x|)^{2} d x} .
$$

Before going on, we remark that inside $B$ one has that $\Xi_{i}=\xi_{i}$ is an eigenfunction for $\mu_{2}(B)$, so if $\Omega=B$ then (4.2) is an equality for all $i=1, \ldots, N$, and then also (4.3) is an equality. Namely,

$$
\mu_{2}(B)=\frac{\int_{B} \phi_{N}^{\prime}(|x|)^{2}+(N-1) \frac{\phi_{N}(|x|)^{2}}{|x|^{2}} d x}{\int_{B} \phi_{N}(|x|)^{2} d x} .
$$

Using the monotone behaviour of $\phi_{N}$ and the fact that $|\Omega|=|B|$, we can easily estimate the denominator as follows

$$
\int_{\Omega} \phi_{N}(|x|)^{2} d x \geq \int_{B} \phi_{N}(|x|)^{2} d x .
$$

On the other hand, we claim that the integrand of the numerator in (4.3) is strictly decreasing for $|x|$ increasing. Indeed, by making use of (2.3), this immediately reduces to check that for all $t>0$ one has

$$
\left(\phi_{N}^{\prime}(t)-\frac{\phi_{N}(t)}{t}\right)^{2}>-\frac{\mu_{2}(B) t \phi_{N}(t) \phi^{\prime}(t)_{N}}{N-1},
$$

which in turn is clearly true since $\phi_{N}>0, \phi_{N}^{\prime} \geq 0$ on $(0,+\infty)$. Therefore,

$$
\int_{\Omega} \phi_{N}^{\prime}(|x|)^{2}+(N-1) \frac{\phi_{N}(|x|)^{2}}{|x|^{2}} d x \leq \int_{B} \phi_{N}^{\prime}(|x|)^{2}+(N-1) \frac{\phi_{N}(|x|)^{2}}{|x|^{2}} d x .
$$

Finally, collecting the estimates (4.5) and (4.6), and using (4.3) and (4.4), we have proven

$$
\begin{aligned}
\mu_{2}(\Omega) \leq & \frac{\int_{\Omega} \phi_{N}^{\prime}(|x|)^{2}+(N-1) \frac{\phi_{N}(|x|)^{2}}{|x|^{2}} d x}{\int_{\Omega} \phi_{N}(|x|)^{2} d x} \\
& \leq \frac{\int_{B}\left[\phi_{N}^{\prime}(|x|)^{2}+(N-1) \phi_{N}(|x|)^{2} /|x|^{2}\right] d x}{\int_{B} \phi_{N}(|x|)^{2} d x}=\mu_{2}(B),
\end{aligned}
$$

thus concluding the proof of the Szegö-Weinberger inequality. Also the equality case follows, since both (4.5) and (4.6) are strict unless $\Omega$ is a ball. 
We now introduce the Szegö-Weinberger deficit, defined as

$$
S W(\Omega):=1-\frac{|\Omega|^{2 / N} \mu_{2}(\Omega)}{|B|^{2 / N} \mu_{2}(B)} .
$$

The main result of this section is the following stability estimate.

Theorem 4.1. For every $\Omega \subset \mathbb{R}^{N}$ open Lipschitz set, we have

$$
S W(\Omega) \geq c_{N} \mathcal{A}(\Omega)^{2} .
$$

Equivalently, the following quantitative form of the Szegö-Weinberger inequality holds,

$$
|B|^{2 / N} \mu_{2}(B)\left(1-c_{N} \mathcal{A}(\Omega)^{2}\right) \geq|\Omega|^{2 / N} \mu_{2}(\Omega),
$$

where $B$ is any ball and $c_{N}$ is a small constant which depends only on the dimension $N$.

Proof. By the scaling invariance of the problem, to show (4.8) we can take a ball $B$ such that $|B|=|\Omega|$, and we can translate it in such a way that (4.3) and (4.4) hold. By definition of asymmetry, one has

$$
\mathcal{A}(\Omega) \leq \alpha:=\frac{|\Omega \Delta B|}{|\Omega|} .
$$

We start observing that, with the same notations as before, (4.3) and (4.4) imply that

$$
\begin{aligned}
& \mu_{2}(B) \int_{B} \phi_{N}(|x|)^{2} d x-\mu_{2}(\Omega) \int_{\Omega} \phi_{N}(|x|)^{2} d x \geq \\
& \int_{B} \phi_{N}^{\prime}(|x|)^{2}+(N-1) \frac{\phi_{N}(|x|)^{2}}{|x|^{2}} d x-\int_{\Omega} \phi_{N}^{\prime}(|x|)^{2}+(N-1) \frac{\phi_{N}(|x|)^{2}}{|x|^{2}} d x .
\end{aligned}
$$

While inequality (4.6) only ensures that the last quantity is positive, our main scope now is to refine that estimate, so to get a quantitative lower bound. In order to do so, we can start determining the "worst case". In fact, let us call, as in Figure $1, B_{1}$ and $B_{2}$ two balls, concentric with $B$ and with radii $r_{1}<r<r_{2}$, in such a way that

$$
|\Omega \cap B|=\left|B_{1}\right|=\omega_{N} r_{1}^{N}, \quad|\Omega \backslash B|=\left|B_{2} \backslash B\right|=\omega_{N}\left(r_{2}^{N}-r^{N}\right),
$$

so that by construction one has

$$
\frac{r^{N}-r_{1}^{N}}{r^{N}}=\frac{\alpha}{2}=\frac{r_{2}^{N}-r^{N}}{r^{N}}
$$

Recalling now that the function

$$
|x| \mapsto \phi_{N}^{\prime}(|x|)^{2}+(N-1) \frac{\phi_{N}(|x|)^{2}}{|x|^{2}}
$$

is strictly decreasing, as we have already checked above, it is immediate to observe that

$$
\int_{\Omega} \phi_{N}^{\prime}(|x|)^{2}+(N-1) \frac{\phi_{N}(|x|)^{2}}{|x|^{2}} d x \leq \int_{B_{1} \cup\left(B_{2} \backslash B\right)} \phi_{N}^{\prime}(|x|)^{2}+(N-1) \frac{\phi_{N}(|x|)^{2}}{|x|^{2}} d x,
$$



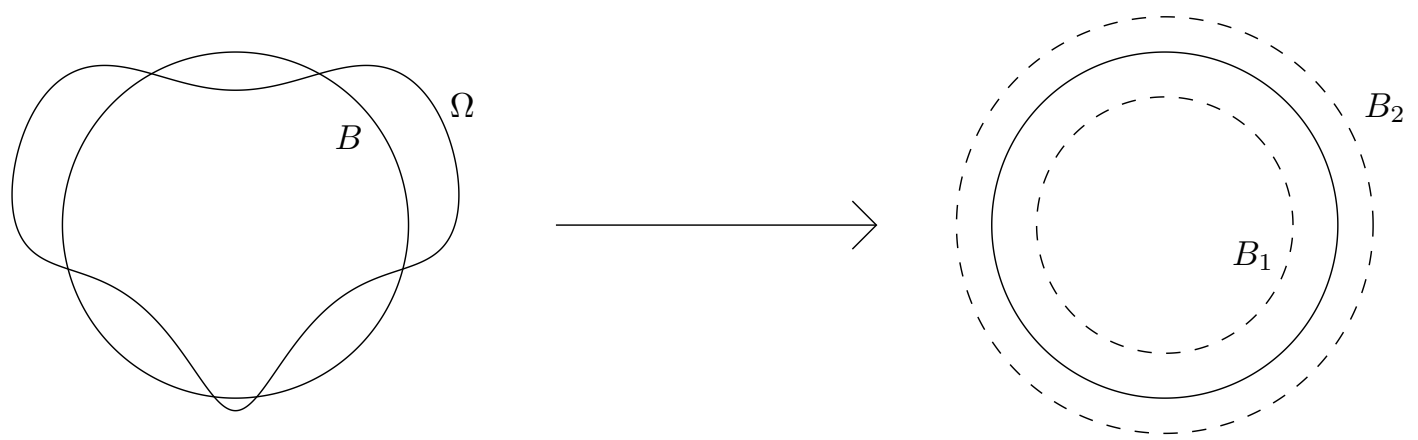

Figure 1. The construction of the proof of Theorem 4.1

so that from (4.10) we can deduce

$$
\begin{aligned}
& \mu_{2}(B) \int_{B} \phi_{N}(|x|)^{2} d x-\mu_{2}(\Omega) \int_{\Omega} \phi_{N}(|x|)^{2} d x \geq \\
& \int_{B \backslash B_{1}} \phi_{N}^{\prime}(|x|)^{2}+(N-1) \frac{\phi_{N}(|x|)^{2}}{|x|^{2}} d x-\int_{B_{2} \backslash B} \phi_{N}^{\prime}(|x|)^{2}+(N-1) \frac{\phi_{N}(|x|)^{2}}{|x|^{2}} d x .
\end{aligned}
$$

Concerning the left-hand side, recalling (4.5) and using (2.2) and (4.1) we have

$$
\int_{\Omega} \phi_{N}(|x|)^{2} d x \geq \int_{B} \phi_{N}(|x|)^{2} d x=r^{2} \int_{\{|y| \leq 1\}}|y|^{2-N} J_{N / 2}\left(\beta_{N / 2,1}|y|\right)^{2} d y=|\Omega|^{2 / N} \eta_{N},
$$

where we have set

$$
\eta_{N}:=\omega_{N}^{-2 / N} \int_{\{|y| \leq 1\}}|y|^{2-N} J_{N / 2}\left(\beta_{N / 2,1}|y|\right)^{2} d y
$$

Notice that $\eta_{N}$ is a constant which only depends on the dimension $N$. Hence, we can estimate the left-hand side of (4.12) as

$$
\begin{aligned}
\mu_{2}(B) \int_{B} \phi_{N}(|x|)^{2} d x-\mu_{2}(\Omega) \int_{\Omega} \phi_{N}(|x|)^{2} d x & \leq\left(\mu_{2}(B)-\mu_{2}(\Omega)\right) \int_{B} \phi_{N}(|x|)^{2} d x \\
& =|\Omega|^{2 / N} \eta_{N}\left(\mu_{2}(B)-\mu_{2}(\Omega)\right) .
\end{aligned}
$$

On the other hand, concerning the right-hand side of (4.12), again recalling that the integrand is strictly decreasing and that $\phi_{N}^{\prime}(t)=0$ for $t \geq r$ we get

$$
\begin{aligned}
\int_{B \backslash B_{1}} \phi_{N}^{\prime}(|x|)^{2}+(N-1) \frac{\phi_{N}(|x|)^{2}}{|x|^{2}} d x & \geq \int_{B \backslash B_{1}}(N-1) \frac{\phi_{N}(r)^{2}}{r^{2}} d x \\
& =\frac{(N-1) \omega_{N} \phi_{N}(r)^{2}}{r^{2}}\left(r^{N}-r_{1}^{N}\right),
\end{aligned}
$$


while

$$
\begin{aligned}
\int_{B_{2} \backslash B} \phi_{N}^{\prime}(|x|)^{2}+(N-1) \frac{\phi_{N}(|x|)^{2}}{|x|^{2}} d x=(N-1) \phi_{N}(r)^{2} \int_{B_{2} \backslash B} \frac{1}{|x|^{2}} d x \\
=N \omega_{N}(N-1) \phi_{N}(r)^{2} \int_{r}^{r_{2}} t^{N-3} d x \leq \frac{N \omega_{N} \phi_{N}(r)^{2}}{r}\left(r_{2}^{N-1}-r^{N-1}\right) .
\end{aligned}
$$

Therefore, inserting (4.13), (4.14) and (4.15) into (4.12), we get

$$
|\Omega|^{2 / N}\left(\mu_{2}(B)-\mu_{2}(\Omega)\right) \geq \frac{\omega_{N} \tilde{\eta}_{N}}{r^{N}}\left((N-1)\left(r^{N}-r_{1}^{N}\right)-N\left(r r_{2}^{N-1}-r^{N}\right)\right)
$$

where, using (2.2) again, we have set

$$
\tilde{\eta}_{N}:=\frac{\phi_{N}(r)^{2}}{\eta_{N}} r^{N-2}=\frac{J_{N / 2}\left(\beta_{N / 2,1}\right)^{2}}{\eta_{N}},
$$

which is still a constant depending only on the dimension $N$.

Since $|\Omega|=|B|$, the thesis will follow as soon as we show that

$$
(N-1) \frac{r^{N}-r_{1}^{N}}{r^{N}}-N \frac{r r_{2}^{N-1}-r^{N}}{r^{N}} \geq c_{N} \mathcal{A}(\Omega)^{2} .
$$

But in fact, by the second equality in (4.11), one has

$$
r_{2}=r\left(1+\frac{\alpha}{2}\right)^{\frac{1}{N}},
$$

and since one surely has $\alpha \leq 2$, this implies

$$
r_{2}^{N-1}=r^{N-1}\left(1+\frac{\alpha}{2}\right)^{\frac{N-1}{N}} \leq r^{N-1}\left(1+\frac{N-1}{N} \frac{\alpha}{2}-c_{N} \alpha^{2}\right),
$$

for a strictly positive constant $c_{N}$ depending only on $N$. Using now the first equality in (4.11), a simple calculation gives

$$
\begin{aligned}
(N-1) \frac{r^{N}-r_{1}^{N}}{r^{N}}-N \frac{r r_{2}^{N-1}-r^{N}}{r^{N}} & =(N-1) \frac{\alpha}{2}-N \frac{r_{2}^{N-1}-r^{N-1}}{r^{N-1}} \\
& \geq(N-1) \frac{\alpha}{2}-N\left(\frac{N-1}{N} \frac{\alpha}{2}-c_{N} \alpha^{2}\right) \\
& =N c_{N} \alpha^{2} .
\end{aligned}
$$

Finally, recalling (4.9) we obtain (4.16) and the proof is concluded.

Before concluding this Section, we make some observations on the comparison between eigenvalues of the Laplacian with different boundary conditions: it is well-known that combining the Faber-Krahn inequality together with the Szegö-Weinberger one, we get

$$
\mu_{2}(\Omega) \leq \mu_{2}(B)<\lambda_{1}(B) \leq \lambda_{1}(\Omega),
$$


that is for every domain $\Omega$ we have $\mu_{2}(\Omega)<\lambda_{1}(\Omega)$. The strict inequality for balls $\mu_{2}<\lambda_{1}$ is based on an explicit computation, which actually gives $\mu_{2}(B)=\theta_{N} \lambda_{1}(B)$, for a positive constant $\theta_{N}<1$ depending only on the dimension $N$ and given by

$$
\theta_{N}=\left(\frac{\beta_{N / 2,1}}{j_{N / 2-1,1}}\right)^{2}
$$

where $\beta_{N / 2,1}$ is as before and $j_{N / 2-1,1}$ is the first zero of the Bessel function $J_{N / 2-1}$. Then, we can rephrase everything by saying that

$$
\mu_{2}(\Omega) \leq \theta_{N} \lambda_{1}(\Omega),
$$

and equality sign holds if and only if $\Omega$ is a ball. We can easily derive a stability estimate for this inequality: namely, we have the following.

Corollary 4.2. For every $\Omega \subset \mathbb{R}^{N}$ open bounded set with Lipschitz boundary, we have

$$
|\Omega|^{2 / N}\left(\theta_{N} \lambda_{1}(\Omega)-\mu_{2}(\Omega)\right) \geq c_{N} \mathcal{A}(\Omega)^{2},
$$

for some constant $c_{N}$ depending only on $N$, while $\theta_{N}$ is given by (4.17).

Proof. Let $B$ be a ball such that $|B|=|\Omega|$, then it is enough to observe

$$
\begin{aligned}
\theta_{N} \lambda_{1}(\Omega)-\mu_{2}(\Omega) & =\theta_{N}\left(\lambda_{1}(\Omega)-\lambda_{1}(B)\right)+\theta_{N} \lambda_{1}(B)-\mu_{2}(\Omega) \\
& =\theta_{N}\left(\lambda_{1}(\Omega)-\lambda_{1}(B)\right)+\mu_{2}(B)-\mu_{2}(\Omega) \geq \mu_{2}(B)-\mu_{2}(\Omega),
\end{aligned}
$$

and then apply (4.7).

\section{Decay RATE of NeARLy CIRCUlar ELlipses}

We now turn to the question of sharpness of the exponent 2 for the Fraenkel asymmetry in (4.7). To do so, we have to exhibit a family $\Omega_{\varepsilon}$ of small deformations of a ball, such that $\mathcal{A}\left(\Omega_{\varepsilon}\right) \rightarrow 0$ and

$$
|B|^{2 / N} \mu_{2}(B) \leq|\Omega|^{2 / N} \mu_{2}\left(\Omega_{\varepsilon}\right)+C_{N} \mathcal{A}\left(\Omega_{\varepsilon}\right)^{2}
$$

with a constant $C_{N}$ independent of $\varepsilon$. If this is true, then we have the asymptotic behaviour $|B|^{2 / N} \mu_{2}(B)-\left|\Omega_{\varepsilon}\right|^{2 / N} \mu_{2}\left(\Omega_{\varepsilon}\right)=O\left(\mathcal{A}\left(\Omega_{\varepsilon}\right)^{2}\right)$, thus proving the sharpness of the exponent 2 in the inequality (4.7) for the Szegö-Weinberger deficit.

The easiest deformations that one can consider are ellipsoids $E_{\varepsilon}$ : in this section, we show that this is not the right choice, proving that

$$
|B|^{2 / N} \mu_{2}(B)-\left|E_{\varepsilon}\right|^{2 / N} \mu_{2}\left(E_{\varepsilon}\right) \approx \mathcal{A}\left(E_{\varepsilon}\right) .
$$

At a first sight, this is quite surprising: indeed, nearly spherical ellipsoids prescribe the right decay rate both in the standard isoperimetric inequality and in the Faber-Krahn one.

In what follows, for simplicity we restrict to the case $N=2$. As it will apparent in a while, this is not restrictive and the very same calculations can be performed for every $N$.

Let $D \subset \mathbb{R}^{2}$ be the disk with unit radius and centered at the origin. For every $\varepsilon>0$, we set

$$
\mathcal{M}_{\varepsilon}=\left[\begin{array}{cc}
1+\varepsilon & 0 \\
0 & \frac{1}{1+\varepsilon}
\end{array}\right]
$$


and we consider the ellipse $E_{\varepsilon}=\mathcal{M}_{\varepsilon} D$. The first non trivial Neumann-Laplacian eigenvalue of $E_{\varepsilon}$ is defined through

$$
\mu_{2}\left(E_{\varepsilon}\right)=\min _{v \in \mathrm{W}^{1,2}\left(E_{\varepsilon}\right) \backslash\{0\}, \int_{E_{\varepsilon}} v(x) d x=0} \frac{\int_{E_{\varepsilon}}|\nabla v(x)|^{2} d x}{\int_{E_{\varepsilon}} v(x)^{2} d x} .
$$

Remark 5.1. It is worth stressing here that for the case of an ellipse, the explicit determination of the eigenvalues is quite a tricky fact. Indeed, proceeding as in the case of the disk, that is, introducing elliptic coordinates and separating the variables, we then arrive to a family of ODEs, called Mathieu equations, whose solutions are correspondingly called Mathieu functions (see [13]). Then, in order to determine the eigenvalues, at least in the Dirichlet case, one has to determine the zeros of these functions. Some interesting (though quite involved and not easy to deal with) asymptotic formulas for the eigenvalues in the case of the Dirichlet-Laplacian can be found in [16] (see formula (5.1), for the case of eccentricity $\approx 0)$. In the Neumann case, the situation is even worse, as far as one has to compute the zeros of derivatives of Mathieu functions. This is why we have preferred here to directly give an expansion of the Rayleigh quotient.

In order to obtain an asymptotic expansion of $\mu_{2}\left(E_{\varepsilon}\right)$ as $\varepsilon$ goes to 0 , it is convenient to set the problem in the disk $D$ with the obvious change of variable: more precisely, observe that defining

$$
\mathcal{B}_{\varepsilon}:=\left[\begin{array}{cc}
\frac{1}{(1+\varepsilon)^{2}} & 0 \\
0 & (1+\varepsilon)^{2}
\end{array}\right]
$$

we have

$$
\mu_{2}\left(E_{\varepsilon}\right)=\min _{u \in \mathrm{W}^{1,2}(D) \backslash\{0\}, \int_{D} u d x=0} \frac{\int_{D}\left\langle\mathcal{B}_{\varepsilon} \nabla u(x), \nabla u(x)\right\rangle d x}{\int_{D} u(x)^{2} d x} .
$$

We can further impose the normalization condition $\int_{D} u(x)^{2} d x=1$, without affecting the minimization problem. Let us set for simplicity

$$
\mathcal{S}(D)=\left\{u \in \mathrm{W}^{1,2}(D) \backslash\{0\}: \int_{D} u(x) d x=0, \int_{D} u(x)^{2} d x=1\right\},
$$

so that

$$
\mu_{2}\left(E_{\varepsilon}\right)=\min _{u \in \mathcal{S}(D)} \int_{D}\left\langle\mathcal{B}_{\varepsilon} \nabla u(x), \nabla u(x)\right\rangle d x
$$

and, at least formally

$$
\begin{aligned}
\int_{D}\left\langle\mathcal{B}_{\varepsilon} \nabla u(x), \nabla u(x)\right\rangle d x & =\int_{D}|\nabla u(x)|^{2} d x+2 \varepsilon \int_{D}\left[\left|\partial_{x_{2}} u(x)\right|^{2}-\left|\partial_{x_{1}} u(x)\right|^{2}\right] d x \\
& +\varepsilon^{2} \int_{D}\left[3\left|\partial_{x_{2}} u(x)\right|^{2}+\left|\partial_{x_{1}} u(x)\right|^{2}\right] d x+o\left(\varepsilon^{2}\right) .
\end{aligned}
$$

The first order term in (5.1) can have a sign: in particular, take the two eigenfunctions corresponding to $\mu_{2}(D)$

$$
\xi_{2}(x)=c J_{1}\left(\beta_{1,1}|x|\right) \frac{x_{1}}{|x|} \quad \text { and } \quad \xi_{3}(x)=c J_{1}\left(\beta_{1,1}|x|\right) \frac{x_{2}}{|x|},
$$


being $c$ the renormalization constant so that $\xi_{2}, \xi_{3} \in \mathcal{S}(D)$. Since $\xi_{2}$ and $\xi_{3}$ are eigenfunctions for $\mu_{2}(D)$, they minimize the leading term in (5.1), while for the first order we have

$$
\int_{D}\left[\left|\partial_{x_{2}} \xi_{2}(x)\right|^{2}-\left|\partial_{x_{1}} \xi_{2}(x)\right|^{2}\right] d x=-\int_{D}\left[\left|\partial_{x_{2}} \xi_{3}(x)\right|^{2}-\left|\partial_{x_{1}} \xi_{3}(x)\right|^{2}\right] d x
$$

and an explicit computation gives

$$
\int_{D}\left[\left|\partial_{x_{2}} \xi_{2}(x)\right|^{2}-\left|\partial_{x_{1}} \xi_{2}(x)\right|^{2}\right] d x<0
$$

This implies that

$$
\mu_{2}\left(E_{\varepsilon}\right)-\mu_{2}(D)=O(\varepsilon)=O\left(\mathcal{A}\left(E_{\varepsilon}\right)\right),
$$

thus showing a linear decay rate in the Szegö-Weinberger inequality. Hence, the ellipsoids do not show the optimality of the exponent 2 in (4.7).

Remark 5.2. Observe that the first order negative term is given by the eigenfuction $\xi_{2}$, corresponding to the direction $x_{1}$ along which the disk has been stretched: the fact that $\mu_{2}(D)-\mu\left(E_{\varepsilon}\right) \approx \varepsilon$ should not be surprising, since $\mu_{2}(D)$ is a multiple eigenvalue, so that the function

$$
\Omega \mapsto \mu_{2}(\Omega),
$$

is not differentiable at the maximum point $D$ (see [9, Chapter 5$]$ ). In a very rough way, we could say that at $D$ the superdifferential of $\mu_{2}(\cdot)$ is a proper set, not just a singleton.

\section{Sharpness of the quantitative Szegö-Weinberger inequality}

In this section, we will show the sharpness of the exponent 2 in the Szegö-Weinberger inequality (4.7). To do so, we will exhibit a family $D_{\varepsilon}$ of sets approaching the disk $D$ of unit radius in such a way that

$$
\mathcal{A}\left(D_{\varepsilon}\right) \approx \frac{\left|D_{\varepsilon} \Delta D\right|}{|D|} \approx \varepsilon, \quad \quad S W\left(D_{\varepsilon}\right) \approx \varepsilon^{2} .
$$

In our construction, we will work for simplicity in dimension $N=2$, but the same argument could be generalized to any dimension $N \geq 2$. Since the whole construction is quite complicate, we will divide this section in various subsections.

6.1. Preliminaries: setting of the construction and main properties. As in the previous section, $D$ stands for the unit disk and we identify its boundary $\partial D$ with the circle $\mathbb{S}^{1}$. We consider a general nearly circular domain, given in polar coordinates by

$$
D_{\varepsilon}=\{(\varrho, \vartheta): \vartheta \in[0,2 \pi], 0 \leq \varrho \leq 1+\varepsilon \psi(\vartheta)\},
$$

where $\psi \in \mathrm{C}^{\infty}\left(\mathbb{S}^{1}\right)$ is such that $\int_{\mathbb{S}^{1}} \psi=0$, thus giving

$$
\left|D_{\varepsilon}\right|-|D| \lesssim \varepsilon^{2} \text {. }
$$

More precisely, we fix an angle $\vartheta_{0} \in(0, \pi / 4)$ and we take a function $\psi$ whose support is given by

$$
\operatorname{supp}(\psi)=\left[-\vartheta_{0}, \vartheta_{0}\right] \cup\left[\pi-\vartheta_{0}, \pi+\vartheta_{0}\right],
$$




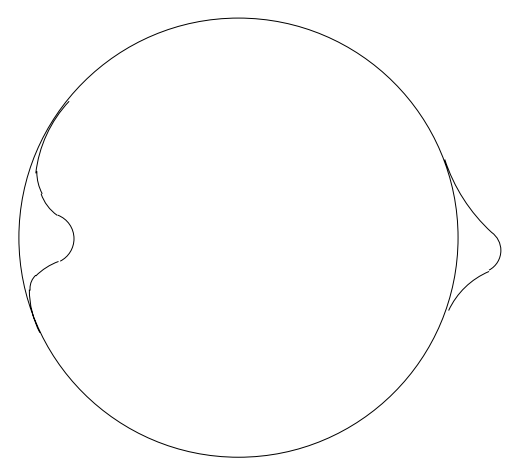

Figure 2. The set $D_{\varepsilon}$

and such that, for any $\vartheta \in\left(-\vartheta_{0}, \vartheta_{0}\right)$,

$$
\psi(\vartheta)>0, \quad \psi(\vartheta+\pi)=-\psi(\vartheta), \quad \psi(-\vartheta)=\psi(\vartheta) .
$$

Observe that $D_{\varepsilon}$ is invariant for reflections with respect to the $x_{1}$ axis, and looks like the set in Figure 2: also observe that by construction one easily gets that

$$
\mathcal{A}\left(D_{\varepsilon}\right) \approx \frac{\left|D_{\varepsilon} \Delta D\right|}{|D|} \approx \varepsilon .
$$

Let us fix now an eigenfunction $u_{\varepsilon}$ for $\mu_{2}\left(D_{\varepsilon}\right)$, normalized in such a way that

$$
\int_{D_{\varepsilon}} u_{\varepsilon}^{2}(x) d x=1, \quad \int_{D_{\varepsilon}}\left|\nabla u_{\varepsilon}(x)\right|^{2} d x=\mu_{2}\left(D_{\varepsilon}\right) .
$$

Remark 6.1. Thanks to the fact that $\partial D_{\varepsilon}$ is of class $\mathrm{C}^{\infty}$, we obtain that $u_{\varepsilon} \in \mathrm{C}^{\infty}\left(\overline{D_{\varepsilon}}\right)$. Moreover, the domains $D_{\varepsilon}$ are uniformly of class $\mathrm{C}^{k}$, for every $k \geq 0$, hence we can assume the functions $u_{\varepsilon}$ to satisfy uniform $\mathrm{C}^{k}$ estimates

$$
\left\|u_{\varepsilon}\right\|_{\mathrm{C}^{k}\left(D_{\varepsilon}\right)} \leq H_{k}
$$

for some constants $H_{k} \in \mathbb{R}^{+}$depending only on $k \in \mathbb{N}$.

Since we want to compare $\mu_{2}\left(D_{\varepsilon}\right)$ with $\mu_{2}(D)$, we have to define suitable functions on $D$. To do so, we consider a $\mathrm{C}^{3}$ extension $\widetilde{u}_{\varepsilon}$ of $u_{\varepsilon}$ to the whole $D \cup D_{\varepsilon}$ : we can make this extension in such a way that

$$
\left\|\widetilde{u}_{\varepsilon}\right\|_{C^{3}\left(D \cup D_{\varepsilon}\right)} \leq K\left\|u_{\varepsilon}\right\|_{C^{3}\left(D_{\varepsilon}\right)} .
$$

In particular, since $\int_{D_{\varepsilon}} u_{\varepsilon}=0$ and $\left|D \backslash D_{\varepsilon}\right|=\left|D_{\varepsilon} \backslash D\right|=O(\varepsilon)$, we have

$$
\delta:=\frac{1}{|D|} \int_{D} \widetilde{u}_{\varepsilon}(x) d x=\frac{1}{|D|}\left(\int_{D \backslash D_{\varepsilon}} \widetilde{u}_{\varepsilon}(x) d x-\int_{D_{\varepsilon} \backslash D} u_{\varepsilon}(x) d x\right)=O(\varepsilon),
$$

because both the integrals are performed on a set of area $\approx \varepsilon$ and the integrands satisfy global $L^{\infty}$ estimates, uniformly in $\varepsilon$, thanks to (6.6) and (6.5). 
We now come to define an admissible function for the Rayleigh quotient defining $\mu_{2}(D)$ : we set

$$
v_{\varepsilon}:=\widetilde{u}_{\varepsilon} \cdot 1_{D}-\delta
$$

and we immediately notice that

$$
\left\|v_{\varepsilon}\right\|_{\mathrm{C}^{3}\left(D_{\varepsilon}\right)} \leq K
$$

thanks to $(6.5),(6.6)$ and (6.7). In words, $v_{\varepsilon}$ is the original eigenfunction $u_{\varepsilon}$ extended to the whole $D \cup D_{\varepsilon}$, then restricted to $D$ and finally vertically translated in order to satisfy the zero-mean condition. By the definition (6.8), and recalling (6.7), we immediately observe that

$$
\begin{aligned}
\left|\int_{D} v_{\varepsilon}^{2}-\widetilde{u}_{\varepsilon}^{2}\right| & =\left|\int_{D}\left(\frac{1}{|D|^{2}}\left(\int_{D} \widetilde{u}_{\varepsilon}\right)^{2}-\frac{2}{|D|}\left(\int_{D} \widetilde{u}_{\varepsilon}\right) \widetilde{u}_{\varepsilon}\right)\right| \\
& =\frac{1}{|D|}\left(\int_{D} \widetilde{u}_{\varepsilon}\right)^{2} \leq K \varepsilon^{2} .
\end{aligned}
$$

Moreover, still using the definition (6.8), and the fact that $\widetilde{u}_{\varepsilon}$ satisfies (6.6) and coincides with $u_{\varepsilon}$ on $D \cap D_{\varepsilon}$, it is also

$$
\left|\int_{D \backslash D_{\varepsilon}} v_{\varepsilon}^{2}-\widetilde{u}_{\varepsilon}^{2}\right| \leq \int_{D \backslash D_{\varepsilon}}\left|v_{\varepsilon}^{2}-\widetilde{u}_{\varepsilon}^{2}\right| \leq K \int_{D \backslash D_{\varepsilon}}\left|v_{\varepsilon}-\widetilde{u}_{\varepsilon}\right|=K \frac{\left|D \backslash D_{\varepsilon}\right|}{|D|} \int_{D} \widetilde{u}_{\varepsilon} \leq K \varepsilon^{2} .
$$

By (6.10) and (6.11), also recalling (6.4), we can thus estimate $\mu_{2}(D)$ from above as follows

$$
\begin{aligned}
\mu_{2}(D) & \leq \frac{\int_{D}\left|\nabla v_{\varepsilon}(x)\right|^{2} d x}{\int_{D} v_{\varepsilon}(x)^{2} d x} \leq \frac{\int_{D \cap D_{\varepsilon}}\left|\nabla u_{\varepsilon}(x)\right|^{2} d x+\int_{D \backslash D_{\varepsilon}}\left|\nabla v_{\varepsilon}(x)\right|^{2} d x}{\int_{D \cap D_{\varepsilon}} u_{\varepsilon}(x)^{2} d x+\int_{D \backslash D_{\varepsilon}} \widetilde{u}_{\varepsilon}(x)^{2} d x-K \varepsilon^{2}} \\
& \leq \frac{\mu_{2}\left(D_{\varepsilon}\right)+\int_{D \backslash D_{\varepsilon}}\left|\nabla v_{\varepsilon}(x)\right|^{2} d x-\int_{D_{\varepsilon} \backslash D}\left|\nabla u_{\varepsilon}(x)\right|^{2} d x}{1+\int_{D \backslash D_{\varepsilon}} v_{\varepsilon}(x)^{2} d x-\int_{D_{\varepsilon} \backslash D} u_{\varepsilon}(x)^{2} d x-K \varepsilon^{2}} .
\end{aligned}
$$

Introducing the two error terms

$$
R_{1}(\varepsilon)=\int_{D \backslash D_{\varepsilon}}\left|\nabla v_{\varepsilon}\right|^{2}-\int_{D_{\varepsilon} \backslash D}\left|\nabla u_{\varepsilon}\right|^{2} \quad \text { and } \quad R_{2}(\varepsilon)=\int_{D \backslash D_{\varepsilon}} v_{\varepsilon}^{2}-\int_{D_{\varepsilon} \backslash D} u_{\varepsilon}^{2},
$$

we thus have obtained the following estimate for $\mu_{2}(D)$

$$
\mu_{2}(D) \leq \frac{\mu_{2}\left(D_{\varepsilon}\right)+R_{1}(\varepsilon)}{1+R_{2}(\varepsilon)-K \varepsilon^{2}}
$$

We notice that thanks to the uniform estimates (6.5) with $k=0,1$, it is immediate to estimate

$$
\left|R_{1}(\varepsilon)\right| \leq K \varepsilon, \quad\left|R_{2}(\varepsilon)\right| \leq K \varepsilon,
$$

which inserted in (6.12) gives the easy estimate

$$
\mu_{2}(D) \leq \mu_{2}\left(D_{\varepsilon}\right)+K \varepsilon .
$$


More generally, we have the following simple but important fact: each estimate on the error terms $R_{1}$ and $R_{2}$ automatically translates into an estimate of the same order for the deficit. Let us state precisely this observation, whose proof is immediate from (6.12).

Lemma 6.2. Let $\omega:[0,1] \rightarrow \mathbb{R}^{+}$be a continuous function with $t^{2} / K \leq \omega(t) \leq K t$. If there exists a constant $C>0$ such that

$$
\left|R_{1}(\varepsilon)\right| \leq C \omega(\varepsilon) \quad \text { and } \quad\left|R_{2}(\varepsilon)\right| \leq C \omega(\varepsilon),
$$

then there exists a constant $C^{\prime}$ such that

$$
\mu_{2}(D) \leq \mu_{2}\left(D_{\varepsilon}\right)+C^{\prime} \omega(\varepsilon)
$$

for every sufficiently small $\varepsilon>0$.

Keeping in mind Theorem 4.1, (6.3) and (6.1), we know that

$$
S W\left(D_{\varepsilon}\right) \approx \mu_{2}(D)-\mu_{2}\left(D_{\varepsilon}\right),
$$

hence to conclude the optimality of the exponent 2 in (4.7) one would like to prove (6.14) with $\omega(t)=t^{2}$.

6.2. First step: some heuristics and a "toy proof". In this subsection, we briefly present the "toy scheme of proof" in order to introduce the main ideas that will give us the searched result. Then, in next subsections, we will give the formal proof. We will focus on the term $R_{1}(\varepsilon)$, but one can easily see that everything works exactly in the same way for $R_{2}(\varepsilon)$.

Let us start observing that

$$
\int_{D_{\varepsilon} \backslash D}\left|\nabla u_{\varepsilon}(x)\right|^{2} d x=\int_{-\vartheta_{0}}^{\vartheta_{0}} \int_{1}^{1+\varepsilon \psi(\vartheta)}\left(\left(\partial_{\varrho} u_{\varepsilon}\right)^{2}+\frac{1}{\varrho^{2}}\left(\partial_{\vartheta} u_{\varepsilon}\right)^{2}\right) \varrho d \varrho d \vartheta
$$

and

$$
\int_{D \backslash D_{\varepsilon}}\left|\nabla v_{\varepsilon}(x)\right|^{2} d x=\int_{\pi-\vartheta_{0}}^{\pi+\vartheta_{0}} \int_{1+\varepsilon \psi(\vartheta)}^{1}\left(\left(\partial_{\varrho} v_{\varepsilon}\right)^{2}+\frac{1}{\varrho^{2}}\left(\partial_{\vartheta} v_{\varepsilon}\right)^{2}\right) \varrho d \varrho d \vartheta .
$$

Using the fact that $\partial_{\varrho} u_{\varepsilon}(\vartheta, \varrho)=O(\varepsilon)$ and $\partial_{\varrho} v_{\varepsilon}(\vartheta, \varrho)=O(\varepsilon)$ for $\varrho=1+O(\varepsilon)$ (which in turn is an immediate consequence of the geometry, together with (6.5) for $k=2)$, and the fact that $\left|D \backslash D_{\varepsilon}\right|=\left|D_{\varepsilon} \backslash D\right| \approx \varepsilon$, one can calculate

$$
\begin{aligned}
\int_{D_{\varepsilon} \backslash D}\left|\nabla u_{\varepsilon}(x)\right|^{2} d x & =\int_{-\vartheta_{0}}^{\vartheta_{0}} \int_{1}^{1+\varepsilon \psi(\vartheta)} \frac{1}{\varrho^{2}}\left(\partial_{\vartheta} u_{\varepsilon}\right)^{2} \varrho d \varrho d \vartheta+o\left(\varepsilon^{2}\right) \\
& =\varepsilon \int_{-\vartheta_{0}}^{\vartheta_{0}} \psi(\vartheta)\left(\partial_{\vartheta} u_{\varepsilon}(\vartheta, 1)\right)^{2} d \vartheta+O\left(\varepsilon^{2}\right),
\end{aligned}
$$

and similarly

$$
\begin{aligned}
\int_{D \backslash D_{\varepsilon}}\left|\nabla v_{\varepsilon}(x)\right|^{2} d x & =\int_{\pi-\vartheta_{0}}^{\pi+\vartheta_{0}} \int_{1+\varepsilon \psi(\vartheta)}^{1} \frac{1}{\varrho^{2}}\left(\partial_{\vartheta} v_{\varepsilon}\right)^{2} \varrho d \varrho d \vartheta+o\left(\varepsilon^{2}\right) \\
& =-\varepsilon \int_{\pi-\vartheta_{0}}^{\pi+\vartheta_{0}} \psi(\vartheta)\left(\partial_{\vartheta} v_{\varepsilon}(\vartheta, 1)\right)^{2} d \vartheta+O\left(\varepsilon^{2}\right) .
\end{aligned}
$$


Hence, recalling that by $(6.2)$ one has $\psi(\vartheta+\pi)=-\psi(\vartheta)$, one gets

$$
\begin{aligned}
R_{1}(\varepsilon) & =-\varepsilon \int_{\pi-\vartheta_{0}}^{\pi+\vartheta_{0}} \psi(\vartheta)\left(\partial_{\vartheta} v_{\varepsilon}(\vartheta, 1)\right)^{2} d \vartheta-\varepsilon \int_{-\vartheta_{0}}^{\vartheta_{0}} \psi(\vartheta)\left(\partial_{\vartheta} u_{\varepsilon}(\vartheta, 1)\right)^{2} d \vartheta+O\left(\varepsilon^{2}\right) \\
& =\varepsilon \int_{-\vartheta_{0}}^{\vartheta_{0}} \psi(\vartheta)\left(\partial_{\vartheta} v_{\varepsilon}(\vartheta+\pi, 1)^{2}-\partial_{\vartheta} u_{\varepsilon}(\vartheta, 1)^{2}\right) d \vartheta+O\left(\varepsilon^{2}\right) \\
& =\varepsilon \int_{-\vartheta_{0}}^{\vartheta_{0}} \psi(\vartheta)\left(\partial_{\vartheta} v_{\varepsilon}(\vartheta+\pi, 1)^{2}-\partial_{\vartheta} v_{\varepsilon}(\vartheta, 1)^{2}\right) d \vartheta+O\left(\varepsilon^{2}\right),
\end{aligned}
$$

where the last equality is due to the fact that by definition $u_{\varepsilon}=v_{\varepsilon}$ up to the constant $\delta$ around $(1, \vartheta)$, with $\vartheta \in\left(-\vartheta_{0}, \vartheta_{0}\right)$. The idea now is quite simple: one can guess that $v_{\varepsilon}$ is sufficiently close to an eigenfunction $\bar{u}$ for $\mu_{2}(D)$. For example, imagine that $v_{\varepsilon}$ coincides with $\bar{u}$, up to an error of order $\varepsilon$ : then substituting $v_{\varepsilon}$ with $\bar{u}$ in the above estimate the integral term would disappear, thanks to the symmetries of the eigenfunctions of the disk. This would improve the rate of convergence to 0 of the term $R_{1}(\varepsilon)$ up to an order $\varepsilon^{2}$.

It is important to notice that more generally, by means of (6.15) and Lemma 6.2, we have the following chain of implications

$$
\min _{\xi \in E_{2}}\left\|v_{\varepsilon}-\xi\right\|_{\mathrm{C}^{1}(\bar{D})} \approx \omega(\varepsilon) \Longrightarrow\left|R_{i}(\varepsilon)\right| \lesssim \varepsilon \omega(\varepsilon) \quad \Longrightarrow \quad\left|\mu_{2}(D)-\mu_{2}\left(D_{\varepsilon}\right)\right| \lesssim \varepsilon \omega(\varepsilon)
$$

for every $\omega$ as in Lemma 6.2, where $E_{2}=\left\{a \xi_{2}+b \xi_{3}:(a, b) \in \mathbb{R} \times \mathbb{R}\right\}$ is the eigenspace relative to $\mu_{2}(D)$. As we will see, this observation constitutes the core of our proof of the sharpness of (4.7).

Remark 6.3. At this point, the reason why ellipsoids did not work in Section 5 becomes evident. Indeed, for the ellipsoid $E_{\varepsilon}$ of the previous section, the function $\psi$ satisfies $\psi(\pi+$ $\vartheta)=\psi(\vartheta)$ rather than (6.2). Hence, in (6.15) one would have that the two terms in the last integral add up instead of subtracting, thus giving $R_{1}(\varepsilon) \approx \varepsilon$, which is perfectly consistent with the fact that $\mu_{2}(D)-\mu_{2}\left(E_{\varepsilon}\right) \approx \varepsilon$.

6.3. Towards the proof: technical machinery. The first technical result we need is the following: the convergence of $v_{\varepsilon}$ to the eigenspace $E_{2}$ is quantified in terms of the decay rate of the error terms $R_{1}$ and $R_{2}$.

Lemma 6.4. Let $\omega$ be a function as in Lemma 6.2. Suppose that there exists $C>0$ such that for every $\varepsilon$ small enough, we have

$$
\left|R_{1}(\varepsilon)\right| \leq C \omega(\varepsilon) \quad \text { and } \quad\left|R_{2}(\varepsilon)\right| \leq C \omega(\varepsilon) .
$$

Then there exists an eigenfunction $\xi_{\varepsilon}$ relative to $\mu_{2}(D)$, such that

$$
\left\|v_{\varepsilon}-\xi_{\varepsilon}\right\|_{\mathrm{C}^{1}(\bar{D})} \leq \widetilde{C} \sqrt{\omega(\varepsilon)}
$$

for some constant $\widetilde{C}$ depending on $C$, but not on $\varepsilon$. 
Proof. We will proceed using a spectral decomposition in $L^{2}(D)$ for $v_{\varepsilon}$, namely

$$
v_{\varepsilon}=\sum_{n \geq 2} a_{n}(\varepsilon) \xi_{n}
$$

where $\left\{\xi_{n}\right\}_{n \geq 1}$ are the (renormalized) Neumann eigenfunctions of $D$, forming an orthonormal basis of $L^{2}(D)$, and where $a_{1}(\varepsilon)=0$ because $v_{\varepsilon}$ has zero-mean.

First of all, one can easily calculate

$$
\sum_{n \geq 2} a_{n}(\varepsilon)^{2}-1=\left\|v_{\varepsilon}\right\|_{L^{2}(D)}^{2}-1=\int_{D} v_{\varepsilon}^{2}-\int_{D_{\varepsilon}} u_{\varepsilon}^{2}=\int_{D} v_{\varepsilon}^{2}-\tilde{u}_{\varepsilon}^{2}-\int_{D \backslash D_{\varepsilon}} v_{\varepsilon}^{2}-\tilde{u}_{\varepsilon}^{2}+R_{2}(\varepsilon),
$$

which by (6.16) and recalling (6.10) and (6.11) yields

$$
\left|\sum_{n \geq 2} a_{n}(\varepsilon)^{2}-1\right| \leq C \omega(\varepsilon)+K \varepsilon^{2} \leq C_{1} \omega(\varepsilon) .
$$

Notice now that by the normalization of the eigenfunctions and by definition one has $\int_{D}\left|\nabla \xi_{n}\right|^{2}=\mu_{n}(D)$ for all $n$, from which we get

$$
\begin{aligned}
\mu_{2}\left(D_{\varepsilon}\right) & =\int_{D_{\varepsilon}}\left|\nabla u_{\varepsilon}(x)\right|^{2} d x \\
& =\int_{D}\left|\nabla v_{\varepsilon}(x)\right|^{2} d x+\int_{D_{\varepsilon} \backslash D}\left|\nabla u_{\varepsilon}(x)\right|^{2} d x-\int_{D \backslash D_{\varepsilon}}\left|\nabla v_{\varepsilon}(x)\right|^{2} d x \\
& =\sum_{n \geq 2} a_{n}(\varepsilon)^{2} \mu_{n}(D)-R_{1}(\varepsilon) .
\end{aligned}
$$

Since assumption (6.16) and Lemma 6.2 ensure that $\left|\mu_{2}(D)-\mu_{2}\left(D_{\varepsilon}\right)\right| \leq C^{\prime} \omega(\varepsilon)$, from $(6.18)$ and (6.19) we get

$$
\left|\sum_{n \geq 2} a_{n}(\varepsilon)^{2} \mu_{n}(D)-\mu_{2}(D)\right| \leq\left(C+C^{\prime}\right) \omega(\varepsilon)=C_{2} \omega(\varepsilon) .
$$

Recall now that $\mu_{2}(D)=\mu_{3}(D)<\mu_{4}(D)$. Hence, by (6.20) and (6.18) we have

$$
\begin{aligned}
C_{2} \omega(\varepsilon) & \geq\left|\sum_{n \geq 2} a_{n}(\varepsilon)^{2} \mu_{n}(D)-\mu_{2}(D)\right| \\
& =\left|\mu_{2}(D)\left(\sum_{n \geq 2} a_{n}(\varepsilon)^{2}-1\right)+\sum_{n \geq 4} a_{n}(\varepsilon)^{2}\left(\mu_{n}(D)-\mu_{2}(D)\right)\right| \\
& \geq\left(\mu_{4}(D)-\mu_{2}(D)\right) \sum_{n \geq 4} a_{n}(\varepsilon)^{2}-\mu_{2}(D) C_{1} \omega(\varepsilon),
\end{aligned}
$$

which gives

$$
\sum_{n \geq 4} a_{n}(\varepsilon)^{2} \leq C_{3} \omega(\varepsilon), \quad \text { hence } \quad\left|a_{2}(\varepsilon)^{2}+a_{3}(\varepsilon)^{2}-1\right| \leq C_{4} \omega(\varepsilon),
$$


where the implication comes again from (6.18). Inserting this estimate into (6.20), one derives

$$
\sum_{n \geq 4} a_{n}(\varepsilon)^{2} \mu_{n}(D) \leq C_{5} \omega(\varepsilon)
$$

Finally, this ensures that $v_{\varepsilon}$ is close, in the $\mathrm{W}^{1,2}$ norm, to the function

$$
\varphi:=a_{2}(\varepsilon) \xi_{2}+a_{3}(\varepsilon) \xi_{3},
$$

which in turn is an eigenfunction for $\mu_{2}(D)=\mu_{3}(D)$. In fact,

$$
\begin{aligned}
\left\|v_{\varepsilon}-\varphi\right\|_{\mathrm{W}^{1,2}(D)}^{2} & =\int_{D}\left(v_{\varepsilon}-\varphi\right)^{2}+\left|\nabla\left(v_{\varepsilon}-\varphi\right)\right|^{2}=\int_{D} \sum_{n \geq 4} a_{n}(\varepsilon)^{2}\left(\xi_{n}^{2}+\left|\nabla \xi_{n}\right|^{2}\right) \\
& =\sum_{n \geq 4} a_{n}(\varepsilon)^{2}\left(1+\mu_{n}(D)\right) \leq C_{6} \omega(\varepsilon) .
\end{aligned}
$$

Summarizing, to conclude the proof we only need to replace the $\mathrm{W}^{1,2}$ norm by the $\mathrm{C}^{1}$ norm. To do so, we are going to use the classical elliptic estimates of Calderon-Zygmund type for the Laplace operator (see [7, Chapter 9]).

Let us then start by observing that, in $D \cap D_{\varepsilon}$, one has

$$
-\Delta v_{\varepsilon}=-\Delta u_{\varepsilon}=\mu_{2}\left(D_{\varepsilon}\right) u_{\varepsilon}=\mu_{2}\left(D_{\varepsilon}\right)\left(v_{\varepsilon}+\delta\right),
$$

where $\delta=O(\varepsilon)$ has been defined in (6.7), and then set

$$
f_{\varepsilon}:=\left\{\begin{array}{ll}
\mu_{2}\left(D_{\varepsilon}\right)\left(v_{\varepsilon}+\delta\right) & \text { on } D \cap D_{\varepsilon}, \\
-\Delta v_{\varepsilon} & \text { on } D \backslash D_{\varepsilon},
\end{array} \quad g_{\varepsilon}:=\left\langle\nabla v_{\varepsilon}, \nu_{D}\right\rangle \quad \text { on } \partial D,\right.
$$

where by $\nu_{D}$ we denote the outer normal to $D$ on $\partial D$. Hence, $v_{\varepsilon}$ is by construction the unique solution of the problem

$$
\left\{\begin{aligned}
-\Delta u & =f_{\varepsilon} & & \text { in } D, \\
\left\langle\nabla u, \nu_{D}\right\rangle & = & g_{\varepsilon} & \text { on } \partial D, \\
\int_{\Omega} u(x) d x & = & 0 . &
\end{aligned}\right.
$$

Similarly, if we set $f=\mu_{2}(D) \varphi$, then $\varphi$ is clearly the unique solution of the problem

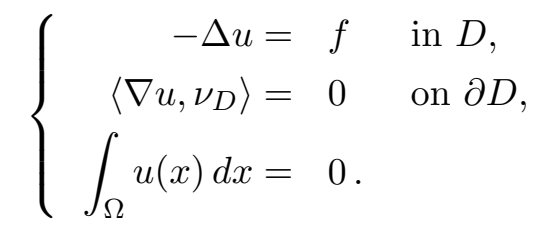

Let us then fix now an exponent $p>N=2$, and keep in mind the global estimate

$$
\left\|D^{2}\left(v_{\varepsilon}-\varphi\right)\right\|_{L^{p}(D)} \leq H\left(\left\|f_{\varepsilon}-f\right\|_{L^{p}(D)}+\left\|g_{\varepsilon}\right\|_{\mathrm{W}^{1-1 / p, p}(\partial D)}+\left\|D\left(v_{\varepsilon}-\varphi\right)\right\|_{L^{p}(D)}\right) .
$$

We start considering the first term of (6.22). By definition, for any $x \in D \cap D_{\varepsilon}$ one has $f_{\varepsilon}(x)=\mu_{2}\left(D_{\varepsilon}\right)\left(v_{\varepsilon}(x)+\delta\right)$. Moreover, for any $x \in D$ there is $\tilde{x} \in D \cap D_{\varepsilon}$ with $|x-\tilde{x}| \leq C \varepsilon$ 
(if $x \in D \cap D_{\varepsilon}$, simply take $\tilde{x}=x$ !). Hence, recalling the uniform estimates (6.9) and (6.7), one can observe that

$$
f_{\varepsilon}(x)=-\Delta v_{\varepsilon}(x)=-\Delta v_{\varepsilon}(\tilde{x})+O(\varepsilon)=\mu_{2}\left(D_{\varepsilon}\right)\left(v_{\varepsilon}(\tilde{x})+\delta\right)+O(\varepsilon)=\mu_{2}\left(D_{\varepsilon}\right) v_{\varepsilon}(x)+O(\varepsilon),
$$

hence

$$
\begin{aligned}
\left\|f_{\varepsilon}-f\right\|_{L^{p}(D)} & =\left\|\mu_{2}\left(D_{\varepsilon}\right) v_{\varepsilon}-\mu_{2}(D) \varphi\right\|_{L^{p}(D)}+O(\varepsilon) \\
& \leq\left|\mu_{2}\left(D_{\varepsilon}\right)-\mu_{2}(D)\right|\left\|v_{\varepsilon}\right\|_{L^{p}(D)}+\mu_{2}(D)\left\|v_{\varepsilon}-\varphi\right\|_{L^{p}(D)}+O(\varepsilon) \\
& \leq C_{7} \omega(\varepsilon)+C_{8} \sqrt{\omega(\varepsilon)}+O(\varepsilon) \leq C_{9} \sqrt{\omega(\varepsilon)},
\end{aligned}
$$

where we have used (6.16) and Lemma 6.2 to estimate $\left|\mu_{2}\left(D_{\varepsilon}\right)-\mu_{2}(D)\right| \leq C^{\prime} \omega(\varepsilon)$, and (6.21) plus a Sobolev embedding to estimate $\left\|v_{\varepsilon}-\varphi\right\|_{L^{p}} \leq C_{8} \sqrt{\omega(\varepsilon)}$.

Concerning the second term in (6.22), one immediately gets

$$
\left\|g_{\varepsilon}\right\|_{\mathrm{W}^{1-1 / p, p}(D)} \leq C \varepsilon
$$

by simple geometric considerations, since $v_{\varepsilon}$ is a $\mathrm{C}^{3}$ extension of $u_{\varepsilon}$ up to an additive constant, and since $u_{\varepsilon}$ satisfies the Neumann boundary condition on $\partial D_{\varepsilon}$.

Finally, let us consider the last term in (6.22). To estimate this, we can use the interpolation inequality

$$
\left\|D\left(v_{\varepsilon}-\varphi\right)\right\|_{L^{p}(D)} \leq C_{\theta}\left\|D\left(v_{\varepsilon}-\varphi\right)\right\|_{L^{2}(D)}+\theta\left\|D^{2}\left(v_{\varepsilon}-\varphi\right)\right\|_{L^{p}(D)},
$$

valid for $\theta \in(0,1)$, and where $C_{\theta}$ is a constant depending on $\theta$ such that $C_{\theta} \rightarrow \infty$ as $\theta$ goes to 0 . By choosing $\theta$ small enough, namely such that $H \theta<1$ being $H$ the constant in (6.22), and by recalling again (6.21), we can insert the estimates (6.23), (6.24) and (6.25) into (6.22) and get

$$
\left\|v_{\varepsilon}-\varphi\right\|_{\mathrm{W}^{2, p}(D)} \leq C_{10} \sqrt{\varepsilon} .
$$

We conclude the validity of (6.17), hence the proof, by a last Sobolev embedding theorem, since $p>2$.

We now rigorously prove that to every improvement on the decay rate of the distance between $v_{\varepsilon}$ and the eigenspace $E_{2}$, there corresponds an improvement (of the same order) on the convergence to 0 of $R_{1}$ and $R_{2}$.

Lemma 6.5. Let $t \mapsto \omega(t)$ satisfies the hypotheses of Lemma 6.2. Suppose that for every $\varepsilon \ll 1$, there exists an eigenfunction $\xi_{\varepsilon}$ for $\mu_{2}(D)$ such that

$$
\left\|v_{\varepsilon}-\xi_{\varepsilon}\right\|_{\mathrm{C}^{1}(\bar{D})} \leq C \sqrt{\omega(\varepsilon)},
$$

for some constant $C$ independent of $\varepsilon$. Then there exists a constant $\widetilde{C}$, still independent of $\varepsilon$, such that

$$
\left|R_{i}(\varepsilon)\right| \leq \widetilde{C} \varepsilon \sqrt{\omega(\varepsilon)}, \quad i=1,2
$$


Proof. Using the uniform estimates (6.5), for any point with polar coordinates $(\varrho, \vartheta) \in$ $D_{\varepsilon} \backslash D$ (hence, with $1 \leq \varrho \leq 1+\varepsilon \psi(\vartheta)$ ), one has

$$
\begin{aligned}
\left|\nabla u_{\varepsilon}(\varrho, \vartheta)\right| & =\left|\nabla u_{\varepsilon}(1+\varepsilon \psi(\vartheta), \vartheta)\right|+O(\varepsilon)=\left|\partial_{\vartheta} u_{\varepsilon}(1+\varepsilon \psi(\vartheta), \vartheta)\right|+O(\varepsilon) \\
& =\left|\partial_{\vartheta} u_{\varepsilon}(1, \vartheta)\right|+O(\varepsilon) .
\end{aligned}
$$

Notice that the second equality comes from the facts that $u_{\varepsilon}$ satisfies a Neumann condition on $\partial D_{\varepsilon}$, and that the normal vector on $\partial D_{\varepsilon}$ is radial up to an error of order $\varepsilon$. Therefore, recalling also that $\left|D_{\varepsilon} \backslash D\right| \approx \varepsilon$, one obtains

$$
\begin{aligned}
\int_{D_{\varepsilon} \backslash D}\left|\nabla u_{\varepsilon}(x)\right|^{2} d x & =\varepsilon \int_{-\vartheta_{0}}^{\vartheta_{0}} \psi(\theta) \partial_{\vartheta} u_{\varepsilon}(1, \theta)^{2} d \vartheta+O\left(\varepsilon^{2}\right) \\
& =\varepsilon \int_{-\vartheta_{0}}^{\vartheta_{0}} \psi(\theta) \partial_{\vartheta} v_{\varepsilon}(1, \theta)^{2} d \vartheta+O\left(\varepsilon^{2}\right),
\end{aligned}
$$

where the last equality comes from the fact that $v_{\varepsilon}=u_{\varepsilon}$ on $D \cap D_{\varepsilon}$ up to an additive constant. In the very same way, recalling that by definition of $v_{\varepsilon}$ one has

$$
\nabla v_{\varepsilon}(1+\varepsilon \psi(\vartheta), \vartheta)=\nabla u_{\varepsilon}(1+\varepsilon \psi(\vartheta), \vartheta)
$$

for all $\vartheta \in\left[\pi-\vartheta_{0}, \pi+\vartheta_{0}\right]$, and that the uniform estimates holds also for $v_{\varepsilon}$ by (6.9), one gets

$$
\int_{D \backslash D_{\varepsilon}}\left|\nabla v_{\varepsilon}(x)\right|^{2} d x=\varepsilon \int_{\pi-\vartheta_{0}}^{\pi+\vartheta_{0}} \psi(\theta) \partial_{\vartheta} v_{\varepsilon}(1, \theta)^{2} d \vartheta+O\left(\varepsilon^{2}\right) .
$$

Let us now apply Lemma 6.4, which is admissible since (6.16) holds by assumption.

Finally, recalling the definition of $R_{1}(\varepsilon)$, from (6.27) and (6.28) and (6.26) one obtains

$$
\begin{aligned}
\left|R_{1}(\varepsilon)\right| & \leq \varepsilon\left|\int_{-\vartheta_{0}}^{\vartheta_{0}} \psi(\vartheta)\left(\left(\partial_{\vartheta} v_{\varepsilon}(1, \vartheta+\pi)\right)^{2}-\left(\partial_{\vartheta} v_{\varepsilon}(1, \vartheta)\right)^{2}\right) d \vartheta\right|+O\left(\varepsilon^{2}\right) \\
& =\varepsilon\left|\int_{-\vartheta_{0}}^{\vartheta_{0}} \psi(\vartheta)\left(\left(\partial_{\vartheta} \xi(1, \vartheta+\pi)\right)^{2}-\left(\partial_{\vartheta} \xi(1, \vartheta)\right)^{2}\right) d \vartheta\right|+C^{\prime} \varepsilon \sqrt{\omega(\varepsilon)}+O\left(\varepsilon^{2}\right) \\
& \leq \widetilde{C} \varepsilon \sqrt{\omega(\varepsilon)},
\end{aligned}
$$

where we used the fact that $\partial_{\vartheta} \xi(\varrho, \vartheta)=-\partial_{\vartheta} \xi(\varrho, \vartheta+\pi)$, true for any eigenfunction $\xi$ for $\mu_{2}(D)$. In the very same way, one can prove that also $\left|R_{2}(\varepsilon)\right| \leq \widetilde{C} \varepsilon \sqrt{\omega(\varepsilon)}$, hence the proof is concluded.

6.4. Sharpness of the exponent 2. We are now ready for the main result of this section. To do so, let us define

$$
\omega_{0}(\varepsilon):=\left|R_{1}(\varepsilon)\right|+\left|R_{2}(\varepsilon)\right| .
$$

This is clearly a continuous function, with $\omega_{0}(\varepsilon) \leq K \varepsilon$ by (6.13), and with $\omega_{0}(\varepsilon) \geq \varepsilon^{2} / K$ by Theorem 4.1. We can then apply Lemma 6.4, which ensures the existence of an eigenfunction $\xi_{\varepsilon}$ for $\mu_{2}(D)$ with the property that

$$
\left\|v_{\varepsilon}-\xi_{\varepsilon}\right\|_{\mathrm{C}^{1}(\bar{D})} \leq C \sqrt{\omega_{0}(\varepsilon)}
$$


for some constant $C$ not depending on $\varepsilon$. Applying then Lemma 6.5, we obtain that

$$
\omega_{0}(\varepsilon)=\left|R_{1}(\varepsilon)\right|+\left|R_{2}(\varepsilon)\right| \leq 2 \widetilde{C} \varepsilon \sqrt{\omega_{0}(\varepsilon)},
$$

being also $\widetilde{C}$ a computable constant, not depending on $\varepsilon$. The previous inequality of course implies that $\omega_{0}(\varepsilon) \leq 4 \widetilde{C}^{2} \varepsilon^{2}$, that is,

$$
\left|R_{1}(\varepsilon)\right| \leq 4 \widetilde{C}^{2} \varepsilon^{2}, \quad\left|R_{2}(\varepsilon)\right| \leq 4 \widetilde{C}^{2} \varepsilon^{2} .
$$

We can then apply Lemma 6.2 with $\omega(\varepsilon)=\varepsilon^{2}$, so to finally obtain $\left|\mu_{2}\left(D_{\varepsilon}\right)-\mu_{2}(D)\right| \approx \varepsilon^{2}$ as desired.

Acknowledgements. The authors warmly acknowledge Nicola Fusco for some fruitful discussions on the topic of this paper, as well as the anonymous referee for having stimulated the improvement of a preliminary version of Section 6 . The authors are also in debt with Mark S. Ashbaugh for some useful historical remarks and for having drawn their attention to the paper [10]. Both authors have been supported by the ERC Advanced Grant n. 226234. The second author has also been supported by the ERC Starting Grant n. 258685, and by the MEC through the grant MTM2008-03541.

\section{REFERENCES}

[1] M. S. Ashbaugh, R. D. Benguria, Isoperimetric inequalities for eigenvalues of the Laplacian. Spectral theory and mathematical physics: a Festschrift in honor of Barry Simon's 60th birthday, 105-139. Proc. Sympos. Pure Math., 76, Part 1, Amer. Math. Soc., Providence, RI, 2007.

[2] T. Bhattacharya, Some observations on the first eigenvalue of the $p$-Laplacian and its connections with asymmetry, Electron. J. Differential Equations, 35 (2001), 15 pp.

[3] T. Bhattacharya, A. Weitsman, Estimates for Green's function in terms of asymmetry, AMS Contemporary Math Series, 221 1999, 31-58.

[4] R. Courant, D. Hilbert, Methods of mathematical physics, Vol. I and II. Interscience Publishers, New York-London 1962.

[5] N. Fusco, F. Maggi, A. Pratelli, The sharp quantitative isoperimetric inequality, Ann. of Math., 168 (2008), 941-980.

[6] N. Fusco, F. Maggi, A. Pratelli, Stability estimates for certain Faber-Krahn, Isocapacitary and Cheeger inequalities, Ann. Sc. Norm. Super. Pisa Cl. Sci., 8 (2009), 51-71.

[7] D. Gilbarg, N. Trudinger, Elliptic Partial Differential Equations of Second Order, 2nd Edition, Springer Verlag, 2001.

[8] A. Henrot, Extremum problems for eigenvalues of elliptic operators. Frontiers in Mathematics. Birkhäuser Verlag, Basel, 2006.

[9] A. Henrot, M. Pierre, Variation et optimisation de formes. (French) [Shape variation and optimization] Une analyse géométrique. [A geometric analysis] Mathématiques \& Applications [Mathematics \& Applications], 48. Springer, Berlin, 2005.

[10] I. Hong, On an inequality concerning the eigenvalue problem of membrane, Kōdai Math. Sem. Rep., 6 (1954), 113-114.

[11] E. T. Kornhauser, I. Stakgold, A variational theorem for $\nabla^{2} u+\lambda u=0$ and its applications, J. Math. Physics, 31 (1952), 45-54.

[12] E. Krahn, Über Minimaleigenschaften der Krugel in drei un mehr Dimensionen, Acta Comm. Univ. Dorpat., A9 (1926), 1-44. 
[13] J. Meixner, F. W. Schäfke, Mathieusche Funktionen und Sphäroidfunktionen mit Anwendungen auf physikalische und technische Probleme. (German). Die Grundlehren der mathematischen Wissenschaften in Einzeldarstellungen mit besonderer Berücksichtigung der Anwendungsgebiete, Band LXXI. Springer-Verlag, Berlin-Göttingen-Heidelberg, 1954.

[14] G. Pólya, On the characteristic frequencies of a symmetric membrane, Math. Zeitschr., 63 (1955), 331-337.

[15] G. Szegö, Inequalities for certain eigenvalues of a membrane of given area, J. Rational Mech. Anal., 3 (1954), 343-356.

[16] B. A. Troesch, H. R. Troesch, Eigenfrequencies of an elliptic membrane, Math. of Computation, 27 (1973), 755-765.

[17] H. F. Weinberger, An isoperimetric inequality for the $N$-dimensional free membrane problem, J. Rational Mech. Anal., 5 (1956), 633-636.

Laboratoire D'Analyse, Topologie, Probabilités UMr6632, Université Aix-Marseille 1, CMI - 39, Rue Frédéric Joliot Curie, 13453 Marseille Cedex 13, France

E-mail address: brasco@cmi.univ-mrs.fr

Dipartimento di Matematica, Università di Pavia "F. Casorati", via Ferrata 1, 27100 Pavia, ITALY

E-mail address: aldo.pratelli@unipv.it 